\title{
(216) Kleopatra, a low density critically rotating M-type asteroid ${ }^{\star, \star \star}$
}

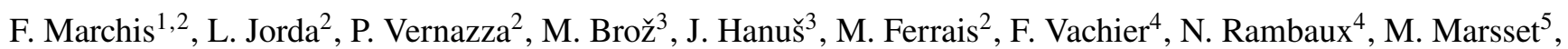 \\ M. Viikinkoski ${ }^{6}$, E. Jehin ${ }^{7}$, S. Benseguane ${ }^{2}$, E. Podlewska-Gaca ${ }^{8}$, B. Carry ${ }^{9}$, A. Drouard ${ }^{2}$, S. Fauvaud ${ }^{10}$, M. Birlan ${ }^{4,11}$, \\ J. Berthier ${ }^{4}$, P. Bartczak ${ }^{8}$, C. Dumas ${ }^{12}$, G. Dudziński ${ }^{8}$, J. Durech ${ }^{3}$, J. Castillo-Rogez ${ }^{13}$, F. Cipriani ${ }^{14}$, F. Colas ${ }^{4}$, \\ R. Fetick ${ }^{2}$, T. Fusco ${ }^{2,15}$, J. Grice ${ }^{9,16}$, A. Kryszczynska ${ }^{8}$, P. Lamy ${ }^{17}$, A. Marciniak ${ }^{8}$, T. Michalowski ${ }^{8}$, P. Michel ${ }^{9}$, \\ M. Pajuelo ${ }^{4,18}$, T. Santana-Ros ${ }^{19,20}$, P. Tanga $^{9}$, A. Vigan ${ }^{2}$, O. Witasse ${ }^{14}$, and B. Yang ${ }^{21}$
}

(Affiliations can be found after the references)

Received 25 March 2021 / Accepted 12 June 2021

\begin{abstract}
Context. The recent estimates of the 3D shape of the M/Xe-type triple asteroid system (216) Kleopatra indicated a density of $\sim 5 \mathrm{~g} \mathrm{~cm}^{-3}$, which is by far the highest for a small Solar System body. Such a high density implies a high metal content as well as a low porosity which is not easy to reconcile with its peculiar "dumbbell" shape.

Aims. Given the unprecedented angular resolution of the VLT/SPHERE/ZIMPOL camera, here, we aim to constrain the mass (via the characterization of the orbits of the moons) and the shape of (216) Kleopatra with high accuracy, hence its density.

Methods. We combined our new VLT/SPHERE observations of (216) Kleopatra recorded during two apparitions in 2017 and 2018 with archival data from the W. M. Keck Observatory, as well as lightcurve, occultation, and delay-Doppler images, to derive a model of its 3D shape using two different algorithms (ADAM, MPCD). Furthermore, an $N$-body dynamical model allowed us to retrieve the orbital elements of the two moons as explained in the accompanying paper.

Results. The shape of (216) Kleopatra is very close to an equilibrium dumbbell figure with two lobes and a thick neck. Its volume equivalent diameter $(118.75 \pm 1.40) \mathrm{km}$ and mass $(2.97 \pm 0.32) \times 10^{18} \mathrm{~kg}$ (i.e., $56 \%$ lower than previously reported) imply a bulk density of $(3.38 \pm 0.50) \mathrm{g} \mathrm{cm}^{-3}$. Such a low density for a supposedly metal-rich body indicates a substantial porosity within the primary. This porous structure along with its near equilibrium shape is compatible with a formation scenario including a giant impact followed by reaccumulation. (216) Kleopatra's current rotation period and dumbbell shape imply that it is in a critically rotating state. The low effective gravity along the equator of the body, together with the equatorial orbits of the moons and possibly rubble-pile structure, opens the possibility that the moons formed via mass shedding.

Conclusions. (216) Kleopatra is a puzzling multiple system due to the unique characteristics of the primary. This system certainly deserves particular attention in the future, with the Extremely Large Telescopes and possibly a dedicated space mission, to decipher its entire formation history.
\end{abstract}

Key words. techniques: high angular resolution - minor planets, asteroids: individual: 216 Kleopatra

\section{Introduction}

Moons around main-belt asteroids have been known to exist since the discovery in 1993 of Dactyl, the companion of (243) Ida (Binzel 1995). Since then, using mostly lightcurve inversion, but also with the use of adaptive optics (AO) on 8-10 m class telescopes and the Hubble Space Telescope (HST), 190 multiple systems have been discovered (Johnston 2021), starting in 1998 with Petit-Prince, around (45) Eugenia (Merline et al. 1999), followed by the discovery of the first triple asteroid (87) Sylvia a few years later (Marchis et al. 2005). Today, about 30 of them have been observed by direct imaging (AO on $8-10 \mathrm{~m}$ class telescopes, HST), providing insights into their formation and evolution (Yang et al. 2016). The images bring indirect information about the interior based on direct information about mass

\footnotetext{
* Reduced images are only available at the CDS via anonymous ftp to cdsarc.u-strasbg. fr $(130.79 .128 .5)$ or via http://cdsarc. u-strasbg.fr/viz-bin/cat/J/A+A/653/A57

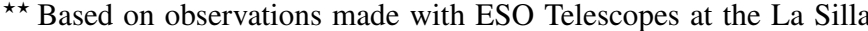
Paranal Observatory under program 199.C-0074 (PI: Vernazza).
}

and volume, and hence density (Margot et al. 2015; Scheeres et al. 2015).

The arrival of a second generation of $\mathrm{AO}$, such as the Spectro-Polarimetric High-contrast Exoplanet Research instrument (SPHERE) at the Very Large Telescope (VLT, Beuzit et al. 2019) and the Gemini Planet Imager (GPI) at GEMINI-South (Macintosh et al. 2014), offers a great opportunity to constrain, via direct imaging, the 3D shape and mass of large multiple asteroid systems where the primary's diameter exceeds $100 \mathrm{~km}$. In 2017 , we started a survey of about forty large $(D \geq 100 \mathrm{~km})$ mainbelt asteroids through a European Southern Observatory (ESO) large programme (id: 199.C-0074, Vernazza et al. 2018), including the following six known multiple systems: (22) Kalliope, (41) Daphne (Carry et al. 2019), (45) Eugenia, (87) Sylvia (Carry et al. 2021), (130) Elektra, and (216) Kleopatra. In addition, our programme allowed the discovery of a new binary asteroid ((31) Euphrosyne, Yang et al. 2020a,b).

Among the six known systems, (216) Kleopatra is of particular interest because of the various density estimates reported for this object, ranging from $\sim 3.6$ to $\sim 5 \mathrm{~g} \mathrm{~cm}^{-3}$ (Descamps et al. 2011; Hanuš et al. 2017; Shepard et al. 2018), and for 
its unique dumbbell shape so far (Ostro et al. 2000; Descamps 2015; Shepard et al. 2018). (216) Kleopatra is a M/Xe-type (DeMeo et al. 2009; Hardersen et al. 2011) triple asteroid system (Descamps et al. 2011) with a high radar albedo (Shepard et al. 2018), likely implying the presence of a substantial fraction of metal at its surface.

Here, we present new AO observations of (216) Kleopatra with VLT/SPHERE/ZIMPOL (Zurich Imaging Polarimeter), which were obtained as part of our ESO large programme (Sect. 2). Combining these new observations, with disk-integrated photometry, stellar occultations, and delayDoppler images, we derived two shape models with the ADAM and MPCD reconstruction methods (Viikinkoski et al. 2015a; Capanna et al. 2012) (Sect. 3). The images were further used to constrain the orbital properties of the two moons and thus constrain the mass of (216) Kleopatra, and hence its density (see Sect. 4 and accompanying paper by Brož et al. 2021). In Sect. 5, we perform a thorough analysis of Kleopatra's shape and propose a formation scenario of this peculiar triple system in Sect. 6 .

\section{Observations and data reduction}

\subsection{Disk-resolved data with SPHERE}

Asteroid (216) Kleopatra was observed at two different epochs in July-August 2017 and December 2018 - January 2019, using ZIMPOL of SPHERE (Thalmann et al. 2008) in the classical imaging mode with the narrow band filter $\left(N \_R\right.$ filter; filter central wavelength $=645.9 \mathrm{~nm}$, width $=56.7 \mathrm{~nm}$ ). The angular size of Kleopatra was in the range of $0.09-0.11^{\prime \prime}$. At the time of the observations, the asteroid was close to an equator-on geometry. Therefore, the SPHERE images of Kleopatra obtained from seven epochs allowed us to reconstruct a reliable 3D shape model with well defined dimensions. The reduced images were further deconvolved with the Mistral algorithm (Fusco et al. 2003), using a parametric point-spread function (Fétick et al. 2019). Table B.1 lists information about the images, while Figs. B.1 and B.2 display all obtained images with SPHERE.

We complemented our dataset with 14 disk-resolved images obtained by the NIRC 2 camera mounted on the W. M. Keck II telescope (Table B.2 and Fig. B.3). These data were already compiled and used for Kleopatra's shape modeling in the study of Hanuš et al. (2017).

The pixel scale of the Zimpol instrument is 3.6 mas, which is almost a factor of three improvement compared to the Keck's NIRC2 camera with a pixel scale of 9.942 mas. We also note that the pixel scale of the VLT/NACO instrument, the decommissioned predecessor of SPHERE, was 13.24 mas.

\subsection{Disk-integrated optical photometry}

We compiled a rich dataset of Kleopatra's disk-integrated optical photometry (180 lightcurves from 15 different apparitions). The oldest data were obtained in 1977 (Scaltriti \& Zappala 1978) and the most recent ones in 2015 (Stéphane Fauvaud). A large fraction of the data spans seven different apparitions in the 1980s (e.g., Pilcher \& Tholen 1982; Weidenschilling et al. 1987). Additionally, we used data from apparitions in 1994 (Fauvaud et al. 2001) and 2006 (Warner 2006). Finally, the largest dataset covering apparitions in 2008, 2010, 2011, and 2013 comes from the SuperWASP survey (Grice et al. 2017). The list of lightcurves is summarized in Table B.3; a subset is then shown in Figs. B.5 and B.6. We did not use any sparse data for their redundancy. Each lightcurve densely sampled the brightness variations for several hours. We treated the lightcurves as relative only, so we normalized the fluxes to unity. We corrected the epochs on lighttime effect. Because each lightcurve is only several hours long, it was not necessary to correct for the phase angle effect.

\subsection{Stellar occultations}

We utilized five stellar occultations of Kleopatra (Table B.4, Herald et al. 2020). While three (from 2009, 2015, and 2016) are of a sufficient quality to be utilized for the shape modeling (i.e., multiple chords sampling the object's projection well), the remaining two (from 1980 and 1991) served as validity checks. We note that the occultation in 1980 is of particular historical interest as two observers independently spotted a 0.9 -second star disappearance too far from the primary to be related with it. At that time, the scientific community was not yet ready to accept the existence of tiny moons around asteroids. This, however, changed in 1994 when the Galileo probe sent images of asteroid (243) Ida with its moon Dactyl. Fortunately, the awareness about these peculiar data persisted. The evident explanation of the data is the occultation by one of Kleopatra's two moons. We list the suspected moon positions in Table 1.

\subsection{Delay-Doppler images}

Delay-Doppler images of Kleopatra were obtained in 2008 and 2013 using the $2380 \mathrm{MHz}$ radar at the Arecibo observatory (Shepard et al. 2018). The 2008 observations were almost equatorial, but with a weak signal-to-noise ratio. Therefore we did not include them in the modeling. Higher quality observations in $2013 \mathrm{had}$ an aspect angle of $-50^{\circ}$ from the equatorial plane. The nominal range resolution is $5.25 \mathrm{~km}$ in range and $10 \mathrm{~Hz}$ in frequency.

\section{3D shape modeling}

\subsection{ADAM shape model}

All-Data Asteroid Modeling (ADAM, Viikinkoski et al. 2015a, and references therein) is an inversion algorithm commonly used for the reconstruction of shape models of asteroids from their combined disk-integrated and disk-resolved data (Viikinkoski et al. 2015b, 2018; Hanuš et al. 2017). The key elements of ADAM are the a priori knowledge of the rotation state (i.e., sidereal rotation period and spin vector orientation) and the existence of disk-resolved data. The former is usually available as convex shape models have been derived for the majority of the largest asteroids (see the Database of Asteroid Models from Inversion Techniques, DAMIT ${ }^{1}$, Ďurech et al. 2010). The most common disk-resolved data are the high-resolution angular images obtained with the 8-10 m class telescopes equipped with AO systems (Keck, VLT, Gemini), but also the more scarce delay-Doppler images (Shepard et al. 2018) or the ALMA interferometry (Viikinkoski et al. 2015b). Finally, stellar occultations can also be considered as disk-resolved data; however, only those with multiple chords with proper timings, sampling the asteroid's on-sky projection well, are useful for constraining the shape.

We applied ADAM to our dataset of 180 optical lightcurves, 14 disk-resolved images from Keck, 55 disk-resolved images from SPHERE, three stellar occultations, and 15 delay-Doppler images from the Arecibo Observatory (Shepard et al. 2018).

https://astro.troja.mff.cuni.cz/projects/damit/ 
Table 1. Positions of Kleopatra's satellites with respect to its photocenter.

\begin{tabular}{|c|c|c|c|}
\hline \multicolumn{4}{|c|}{ AlexHelios } \\
\hline Date & UT & $u[\operatorname{arcsec}]$ & $v[\operatorname{arcsec}]$ \\
\hline 1980-10-10 & 07:00 & 0.2711 & 0.4564 \\
\hline 2008-09-19 & 11:38:00 & -0.18 & 0.35 \\
\hline 2008-09-19 & $11: 51: 00$ & -0.20 & 0.36 \\
\hline 2008-10-05 & 09:13:00 & -0.27 & 0.37 \\
\hline 2008-10-05 & 09:49:00 & -0.29 & 0.39 \\
\hline 2008-10-05 & 10:03:00 & -0.32 & 0.39 \\
\hline 2008-10-09 & $05: 46: 00$ & -0.32 & 0.29 \\
\hline 2017-07-14 & 05:00:59 & -0.4158 & 0.2952 \\
\hline $2017-07-22$ & 04:18:07 & -0.4262 & 0.2444 \\
\hline 2017-07-22 & 05:00:55 & -0.4291 & 0.2614 \\
\hline $2017-08-22$ & $01: 42: 34$ & 0.1843 & -0.3150 \\
\hline 2018-12-10 & $06: 47: 17$ & -0.4894 & -0.0958 \\
\hline 2018-12-19 & 06:45:02 & 0.1871 & -0.2869 \\
\hline $2018-12-22$ & $05: 58: 43$ & -0.2540 & -0.3438 \\
\hline $2018-12-26$ & $08: 14: 42$ & -0.4973 & -0.0428 \\
\hline 2019-01-14 & 04:57:43 & -0.5319 & -0.1177 \\
\hline \multicolumn{4}{|c|}{ CleoSelene } \\
\hline 2008-09-19 & 06:17:00 & -0.25 & 0.50 \\
\hline 2008-09-19 & 08:44:00 & -0.34 & 0.54 \\
\hline 2008-09-19 & 11:38:00 & -0.44 & 0.57 \\
\hline 2008-09-19 & $11: 51: 00$ & -0.44 & 0.57 \\
\hline 2008-09-19 & 12:02:00 & -0.44 & 0.58 \\
\hline 2008-10-05 & 09:13:00 & -0.29 & 0.46 \\
\hline 2008-10-05 & 09:49:00 & -0.31 & 0.47 \\
\hline 2008-10-05 & 10:03:00 & -0.32 & 0.46 \\
\hline 2008-10-09 & 09:36:00 & 0.28 & -0.44 \\
\hline 2017-07-14 & 05:01:00 & 0.3070 & -0.2600 \\
\hline 2017-07-22 & 04:18:07 & -0.2714 & 0.2941 \\
\hline $2017-07-22$ & 05:00:55 & -0.2621 & 0.2963 \\
\hline 2017-08-22 & $01: 42: 34$ & 0.4423 & -0.2401 \\
\hline 2018-12-10 & $06: 47: 17$ & 0.1949 & -0.1505 \\
\hline 2018-12-19 & $06: 45: 02$ & 0.3763 & 0.0929 \\
\hline $2018-12-22$ & $05: 58: 43$ & 0.4555 & -0.0119 \\
\hline $2018-12-26$ & 08:14:42 & 0.3937 & 0.1271 \\
\hline 2019-01-14 & $04: 57: 43$ & -0.1008 & -0.3298 \\
\hline
\end{tabular}

Notes. Uncertainties are approximately 0.01 arcsec. The position from 1980 is based on the stellar occultation and those from 2008 are taken from Descamps et al. (2011).

We used the rotation state (Hanuš et al. 2017) as an initial value for the ADAM modeling with a low shape model resolution (1152 facets) and the octantoid shape parametrization (Viikinkoski et al. 2015a). Then, we increased the shape model resolution (2048 facets) and used the low-resolution model as a starting point for the shape model improvement. We also increased the relative weight of the SPHERE data with respect to other datasets. We show the comparison of the shape model projections with the corresponding SPHERE and Keck/NIRC2 images in Figs. 1 and B.3, and with the stellar occultations in Fig. B.4. The fit to a subset of optical lightcurves is shown in Figs. B.5, B.6. Our solution is robust against variations in data weighting and ADAM regularization functions. We generated several models both with and without delay-Doppler images. The optimization method used for radar images is described in detail in Viikinkoski et al. (2015a). We used the cosine scattering law with constant albedo. By increasing the relative weight of radar data with respect to AO images, the shape solution approaches the shape presented in Shepard et al. (2018). The choice of weights between different data sources is always a somewhat subjective matter. However, in this case, both the coverage and the resolution of $\mathrm{AO}$ images is clearly superior compared to radar images, so it seems prudent that the AO observations from SPHERE are given predominance. The comparison between radar data and the shape model is shown in Fig. B.7.

Our final ADAM shape model fits all datasets sufficiently well. Specifically, we have not identified any substantial disagreement between the model and the data. Considering the superior quality, the resolution, and the coverage of the SPHERE data, the shape model is already well constrained by them. The other data (Keck, occultations, radar) are usually fitted naturally and are mostly complementary.

\subsection{MPCD shape model}

The Multiresolution PhotoClinometry by Deformation (MPCD, Capanna et al. 2012; Jorda et al. 2016) is a 3D shape reconstruction method that utilizes an initial shape model to give a better fit to disk-resolved images. Therefore, our ADAM shape model is further modified by MPCD by fitting solely the high-resolution SPHERE AO data. The MPCD algorithm minimizes the chi-square pixel-to-pixel differences between a set of observed images and the synthetic images built from the shape model for optimization. The reflectance function is Hapke's fiveparameters function with the parameters listed in Descamps et al. (2011). The shape is optimized through shifts of the vertices along the local normal vector. The method goes through several increasing steps of resolutions of both the observed images and shape before converging toward the final optimized model. In the case of (216) Kleopatra, we used the sample of 33 SPHERE images obtained during 11 visits. The shape was reconstructed starting from a decimated ADAM model with only 196 facets and ending with a final model of 3136 facets, after optimization in three levels. Furthermore, we also optimized the Euler angles describing the orientation of the spin pole after noticing unusual systematic residuals between our sets of observed and synthetic images. The shape was reoptimized with this new pole orientation in the same manner to produce the final MPCD model of (216) Kleopatra. As expected, the chi-square between observed and synthetic AO images decreases from 135 (ADAM model) to 50 (MPCD model). A comparison between synthetic SPHERE images generated from the ADAM and MPCD models and an observed image for each visit is shown in Fig. 1. The final MPCD model is shown in Fig. 2 alongside the ADAM model and the radar model of Shepard et al. (2018). The physical properties of those three models are listed in Table 2. The MPCD method also provides an albedo map calculated together with the slope and height errors of each facet from their corresponding average residual pixel values. However, the only significant albedo features are found near limbs and a careful inspection shows that they likely correspond to faint artifacts introduced by the deconvolution process (Fétick et al. 2019) at the edges of the object.

\section{Mass and bulk density}

In the first step, each image obtained with SPHERE/ZIMPOL was further processed to remove the bright halo surrounding Kleopatra, following the procedure described in detail in Yang et al. (2016) and Pajuelo et al. (2018), and shown in Fig. 3. The residual structures after the halo removal were minimized using the processing techniques introduced in Wahhaj et al. (2013), 

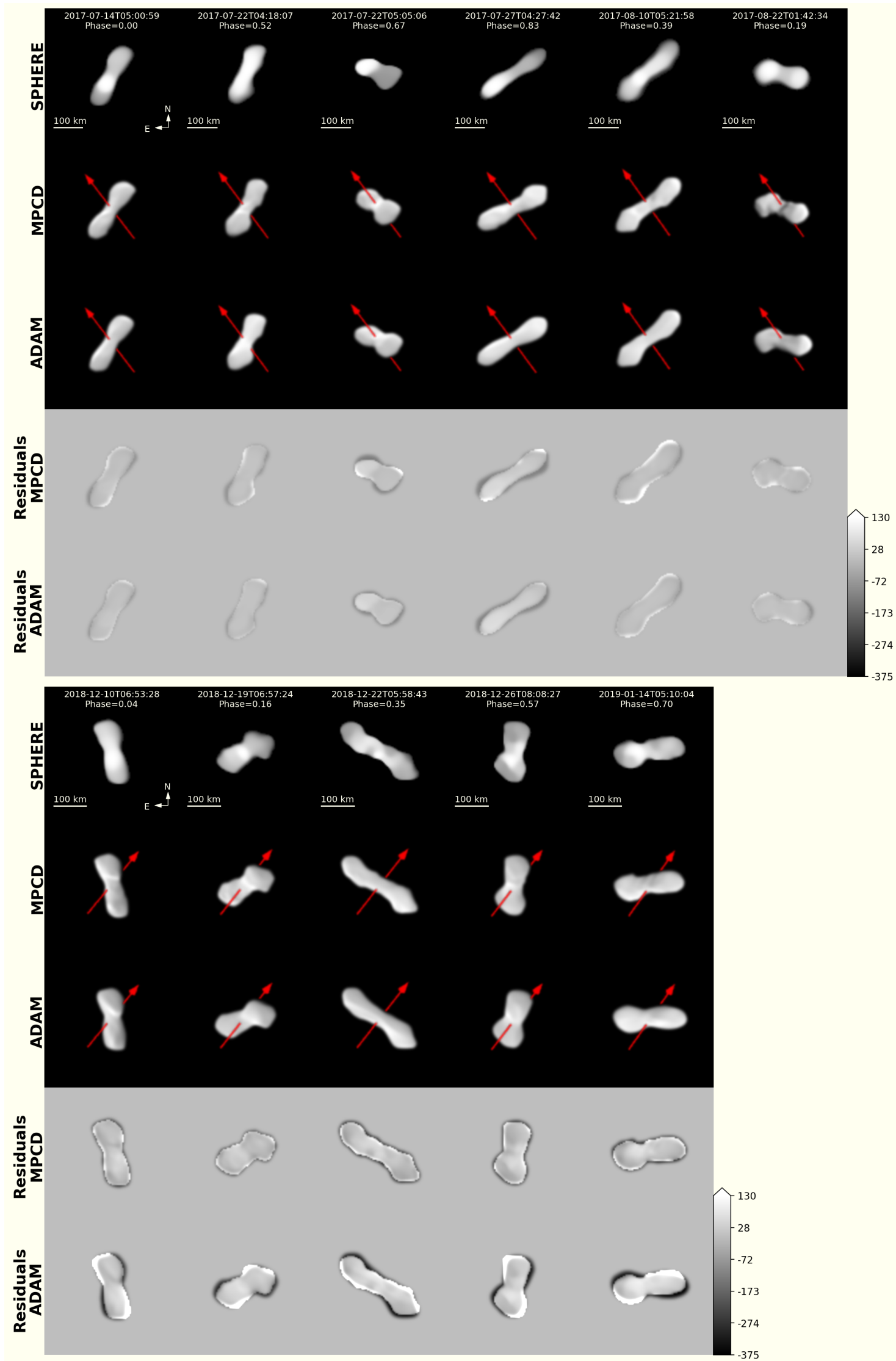

Fig. 1. Comparison between the VLT/SPHERE/ZIMPOL deconvolved images of Kleopatra and the corresponding projections of our MPCD and ADAM shape models. The red arrow indicates the orientation of the spin axis. We used a realistic illumination to highlight the local topography of the model using the OASIS software (Jorda et al. 2010). The residuals of both models are shown in the two bottom rows, more specifically those are chi-square pixel residuals in units of the instrumental noise associated to each pixel (photon and readout noise). 

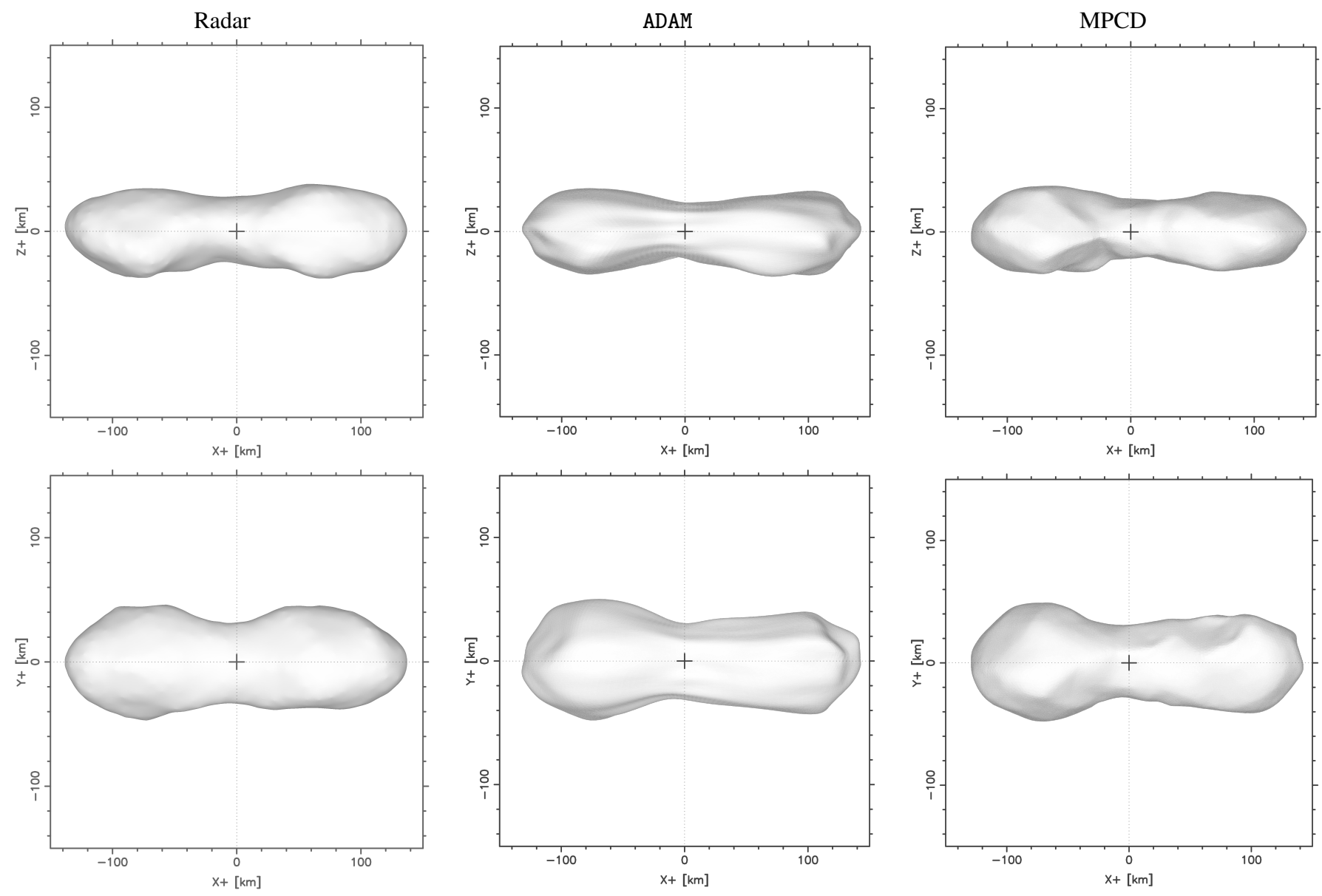

Fig. 2. Rendered views of the radar shape model of Shepard et al. (2018) (left panels), and ADAM and MPCD models (middle and right panels) as seen from the $Y-$ (top panels) and $Z+$ (bottom panels) body axes. The mass deficit or crater is visible on the small lobe of the MPCD model.

Table 2. Physical properties of (216) Kleopatra based on ADAM and MPCD shape modeling of our VLT/SPHERE images.

\begin{tabular}{lccc}
\hline \hline Parameter & S18 & ADAM & MPCD \\
\hline$P(\mathrm{~h})$ & $5.385280(1)$ & $5.385282(1)$ & $5.385282(1)$ \\
$\lambda\left(^{\circ}\right)$ & $74 \pm 2$ & $73.5 \pm 0.5$ & $74.1 \pm 0.5$ \\
$\beta\left({ }^{\circ}\right)$ & $20 \pm 2$ & $20.8 \pm 0.5$ & $21.6 \pm 0.5$ \\
$D(\mathrm{~km})$ & $122 \pm 10$ & $119.3 \pm 2$ & $118.2 \pm 0.8$ \\
$a(\mathrm{~km})$ & $276 \pm 14$ & $270 \pm 4$ & $267 \pm 6$ \\
$b(\mathrm{~km})$ & $94 \pm 5$ & $62 \pm 4$ & $61 \pm 6$ \\
$c(\mathrm{~km})$ & $78 \pm 4$ & $38 \pm 4$ & $48 \pm 6$ \\
$a / b$ & 2.9 & $4.35 \pm 0.3$ & $4.4 \pm 0.4$ \\
$b / c$ & 1.20 & $1.63 \pm 0.2$ & $1.3 \pm 0.2$ \\
$V\left(10^{5} \mathrm{~km}^{3}\right)$ & 9.56 & $8.90 \pm 0.45$ & $8.65 \pm 0.17$ \\
$M\left(10^{18} \mathrm{~kg}\right)$ & $4.64 \pm 0.02$ & $2.97 \pm 0.32$ & $2.97 \pm 0.32$ \\
$\left.\rho(\mathrm{g} \mathrm{cm})^{-3}\right)$ & $4.9 \pm 0.5$ & $3.34 \pm 0.53$ & $3.43 \pm 0.38$ \\
\hline$\chi^{2}$ & 209 & 135 & 50 \\
\hline
\end{tabular}

Notes. The table lists: sidereal rotation period $P$, spin-axis ecliptic J2000 coordinates $\lambda$ and $\beta$, volume-equivalent diameter $D$, dimensions along the major axis $a, b, c$, their ratios $a / b$ and $b / c$, mass $M$ from Brož et al. (2021, accompanying paper), volume $V$, and bulk density $\rho$. Uncertainties correspond to $1 \sigma$ values. The values based on radar data (Shepard et al. 2018) are also reported. The $b$ and $c$ extents reported for the radar model are the maximum extents in those directions. where the radial structures were removed using a running median in a 30 -pixel box in the radial direction and the images were smoothed by convolving a Gaussian function with a FWHM of $\sim 8$ pixels.

In the second step, we measured the relative positions on the plane of the sky between Kleopatra and its satellites. We used the unmodified images (i.e., without halo removal) and we fit both photocenters by a suitable 2D Gaussian (see Carry et al. 2019). The dispersion of the Gaussian function for the moons was chosen conservatively as comparable to the residual (AO-corrected) PSF. The astrometric positions are reported in Table 1. Sometimes, the identification of the two satellites was ambiguous. Nevertheless, it was possible to recover the correct identification later (see Brož et al. 2021). We report the corrected data here.

Furthermore, we estimated the offsets between the photocenter and the center of mass for Kleopatra. Because the central body is so extended and irregular, the offset may reach up to a few milli-arcseconds, as reported in Table 3. We use these offset adjustments for further analysis of orbits because our dynamical model requires the centers of mass, not the centers of light. Alternatively, one can use a relative astrometry of the two moons (second with respect to the first), which is unaffected by these photocenter motions.

Uncertainties in the measurements are approximately 0.01 arcsec, based on repeated and/or close-in-time measurements. As of now, we do not account for the orbital motion 

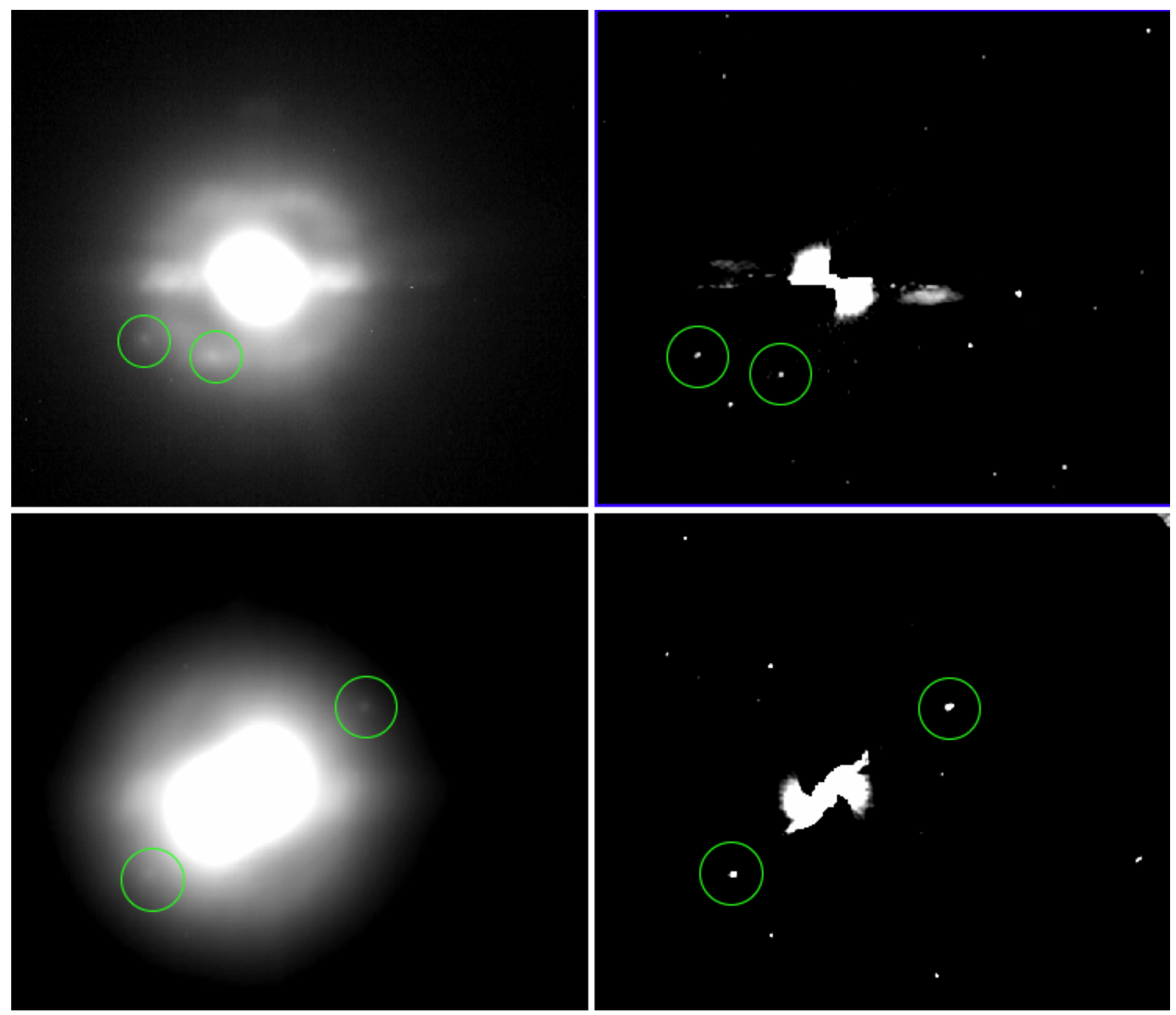

Fig. 3. Processed ZIMPOL images on the left, revealing the presence of the satellites, CleoSelene and AlexHelios around (216) Kleopatra at two epochs (bottom: 2017-07-14, top: 2017-08-22). To reveal the moons which are as faint as the halo (due to imperfect AO correction), we subtracted a rotational average of the image centered on the primary (right image). The circle points to the location of the satellites in the images. The other dots in the images are bad pixels.

of the satellites during five consecutive exposures and we take their average position, although in principle that motion could be detected. It would however require a fitting by an asymmetric PSF, elongated along the orbital motion.

The dynamical model required to interpret the motion of the moons is more complex due to the irregular shape of Kleopatra, and the mutual interactions of the moons and the solar tides. We thus used an advanced $N$-body model with the multipole expansion up to the order of $\ell=10$, which is described in detail in Brož et al. (2021), in order to determine the orbital elements. Our best solution fits the observed positions with a root mean square (RMS) residualss of 17 mas. Most importantly, the phase coverage of new VLT/SPHERE observations allowed us to derive the true periods $P_{1}=(1.822359 \pm 0.004156) \mathrm{d}$ and $P_{2}=(2.745820 \pm 0.004820) \mathrm{d}$, which results in the revised mass $m_{1}=(2.97 \pm 0.32) \times 10^{18} \mathrm{~kg}$ for Kleopatra. This is significantly lower than the previously reported value of $4.64 \times 10^{18} \mathrm{~kg}$ (Descamps et al. 2011). The orbits of both satellites are circular, prograde, and equatorial, similar to most known satellites around large main belt asteroids. (e.g., Marchis et al. 2008; Berthier et al. 2014; Margot et al. 2015; Carry et al. 2019; Yang et al. 2020a).

Taking the average volume of the ADAM and MPCD models (Table 2), the density of Kleopatra amounts to $(3.38 \pm 0.50) \mathrm{g} \mathrm{cm}^{-3}$. A comparison with the previous estimate (3.6 $\mathrm{g} \mathrm{cm}^{-3}$, Descamps et al. 2011) is not pertinent given that both the mass and the volume were revised. This density has important implications for the interpretation of the shape in Sect. 5.

\section{Shape analysis}

\subsection{Lobes interpretation}

A visual inspection of the global shape confirms the presence of two lobes separated by a neck, at the first order similar to the shape derived in Ostro et al. (2000). For this reason, we extracted 

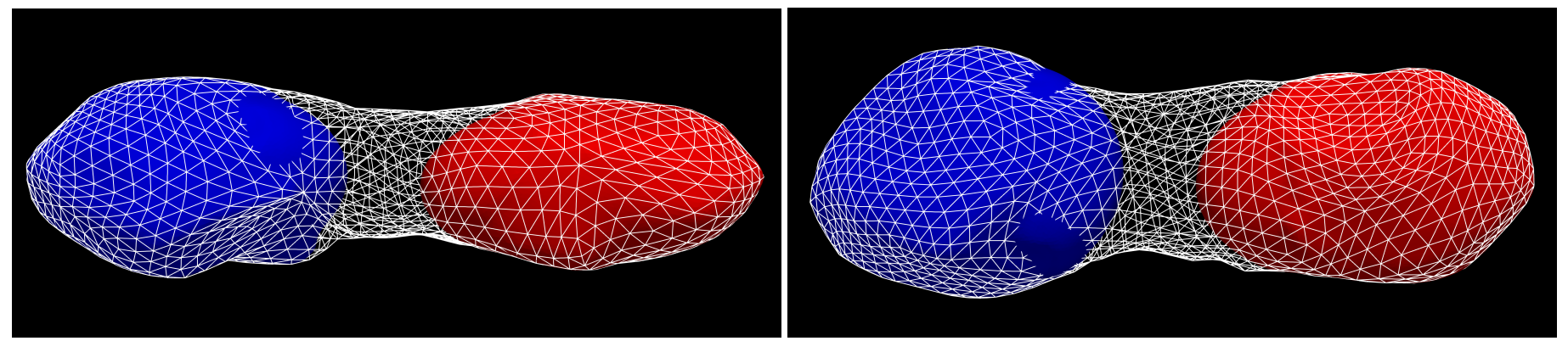

Fig. 4. Views of the two lobes A (blue) and B (red) extracted from the shape model as seen from the $Y+(l e f t)$ and $Z+($ right $)$ axes. The initial shape model is displayed as a wireframe for comparison, allowing one to visualize the neck between the two lobes.

Table 3. Positions of the photocenter minus the center of mass for Kleopatra.

\begin{tabular}{ccrr}
\hline \hline $\mathrm{JD}$ & $\mathrm{UT}$ & $u$ [mas] & $v$ [mas] \\
\hline $2008-09-19$ & $06: 17: 00$ & 0.762 & 0.789 \\
$2008-09-19$ & $08: 44: 00$ & -0.948 & -1.395 \\
$2008-09-19$ & $11: 38: 00$ & 1.178 & 1.069 \\
$2008-09-19$ & $11: 51: 00$ & -1.462 & -0.737 \\
$2008-09-19$ & $12: 02: 00$ & -3.723 & -1.784 \\
$2008-10-05$ & $09: 13: 00$ & 4.586 & 2.911 \\
$2008-10-05$ & $09: 49: 00$ & 4.059 & 1.363 \\
$2008-10-05$ & $10: 03: 00$ & 2.212 & -0.395 \\
$2008-10-09$ & $05: 46: 00$ & 0.548 & -2.190 \\
$2008-10-09$ & $09: 36: 00$ & 6.923 & 5.164 \\
$2017-07-14$ & $05: 01: 00$ & 0.223 & -1.194 \\
$2017-07-22$ & $04: 18: 07$ & 0.609 & 1.934 \\
$2017-07-22$ & $05: 00: 55$ & 0.666 & 1.929 \\
$2017-08-22$ & $01: 42: 34$ & 0.288 & 0.901 \\
$2018-12-10$ & $06: 47: 17$ & -1.674 & -2.402 \\
$2018-12-19$ & $06: 45: 02$ & 0.391 & -3.132 \\
$2018-12-22$ & $05: 58: 43$ & 2.014 & -1.430 \\
$2018-12-26$ & $08: 14: 42$ & -0.848 & 0.791 \\
$2019-01-14$ & $04: 57: 43$ & -0.455 & -1.266 \\
\hline
\end{tabular}

the individual shapes of the two lobes from the MPCD global shape model in order to characterize their physical properties. We used an approach similar to that applied to comet 67P/C-G (Jorda et al. 2016). In this approach, the facets belonging to each lobe are manually selected in the "Meshlab software" (Cignoni et al. 2008). The best-fit ellipsoid of each lobe is then computed by fitting the coordinates of the extracted vertices. Finally, the lobe models are merged with those of the best-fit ellipsoids to compute their closed shapes and volumes (for details on the method, see Jorda et al. 2016). This leads to the individual shapes of the two lobes shown in Fig. 4.

We determined the diameters of the two lobes along the $X$-, $Y$-, and $Z$-axes and along their principal axes of inertia, as well as their volume (summarized in Table 4). It appears that the $X$ - and $Z$ - axes have the same diameters within their error bars. However, the $Y$-axis is significantly different between the two lobes, leading to a volume difference of $16 \%$ in favor of lobe A. Furthermore, both lobes appear highly ellipsoidal, with a deviation between the lobes and their best-fit ellipsoids of only $\sim 2.5-3 \%$, a value comparable to those found for large asteroids with equilibrium shapes, such as 10 Hygiea (Vernazza et al. 2020) and 4 Vesta (Ferrais et al. 2020). The size difference between the two lobes along the $Y$-axis can possibly be explained by a depression
Table 4. Physical parameters (volume and diameters) of the two lobes computed from their reconstructed shape models, as well as geometric parameters (center coordinates, Euler angles, and tilt of the lobes' $Z$-axis with respect to that of the object), resulting from the best-fit ellipsoid of the selected facets of the MPCD shape model belonging to each lobe.

\begin{tabular}{lcc}
\hline \hline Parameter & Lobe A & Lobe B \\
\hline Diameter $a(\mathrm{~km})$ & 118 & 126 \\
Diameter $b(\mathrm{~km})$ & 94 & 79 \\
Diameter $c(\mathrm{~km})$ & 66 & 61 \\
Volume $\left(10^{5} \mathrm{~km}^{3}\right)$ & 4.1 & 3.5 \\
Volume $(\%)$ & 47 & 40 \\
Volume-equiv. diameter $D(\mathrm{~km})$ & 91.9 & 87.1 \\
\hline Center coordinate $X(\mathrm{~km})$ & -69.9 & 79.8 \\
Center coordinate $Y(\mathrm{~km})$ & 0.0 & -0.5 \\
Center coordinate $Z(\mathrm{~km})$ & 0.2 & -0.9 \\
Euler angle $\psi\left({ }^{\circ}\right)$ & 39.0 & -21.7 \\
Euler angle $\theta\left(^{\circ}\right)$ & -5.6 & 5.0 \\
Euler angle $\phi\left(^{\circ}\right)$ & -35.2 & 26.3 \\
$Z$-axis tiltt $\left.{ }^{\circ}\right)$ & 5.6 & 5.0 \\
Ellipsoid fit residuals $(\mathrm{km})$ & 2.2 & 1.8 \\
Ellipsoid fit residuals $(\%)$ & 3.1 & 2.4 \\
\hline
\end{tabular}

observed on lobe B (see Fig. 4), possibly formed by an impact.

As a next step, we computed the length and mean radii of the neck as well as its volume from the parameters of Table 4. Its volume appears to be $13 \%$ of the total volume of the object, whereas the minimum length of the neck along the $X$-axis is $\sim 25 \mathrm{~km}$.

\subsection{Dumbbell interpretation}

It is striking that in Fig. 2, the shape of Kleopatra resembles the "dumb-bell" equilibrium shapes studied by Descamps (2015). To test whether Kleopatra formed at equilibrium, we computed the rms of the deviation between the MPCD shape model of Kleopatra and several shape models of dumbbell equilibrium figures corresponding to different values of the normalized angular velocity $\Omega$ defined by Descamps (2015) as $\omega \sqrt{3 /(4 \pi G \rho)}$, where $\rho$ denotes the bulk density. In order to perform this comparison, we rescaled the equilibrium shapes so that their lengths match that of Kleopatra and we shifted the center of the figure by $-3.8 \mathrm{~km}$ along the $X$-axis. This shift leads to a displacement of the center of mass toward the larger lobe (lobe A), which is coherent if we assume that the two lobes have the same density. The lowest final rms of the distance between the models is equal 

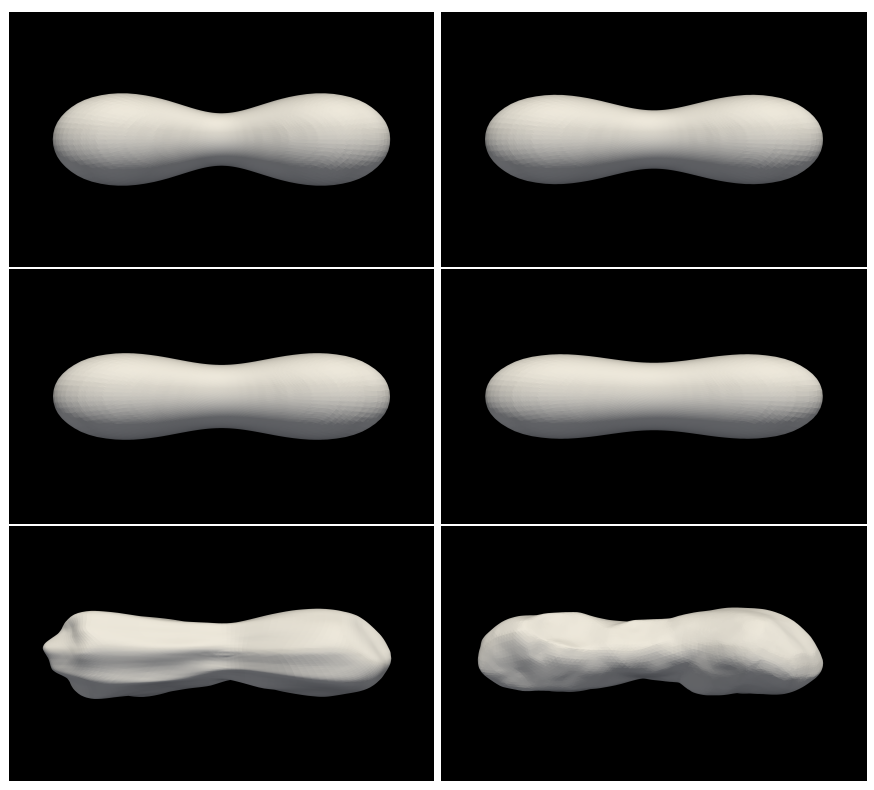

Fig. 5. Views of the dumbbell equilibrium shapes for values of the normalized angular velocity $\Omega$ equal to 0.31 (top left), 0.32 (top right), 0.33 (middle left), and 0.34 (middle right). The two bottom panels show the reconstructed ADAM (left) and MPCD (right) shapes. All shape models are seen from the $Y$ - axis.

to $\sim 3.5 \mathrm{~km}$ for a normalized angular velocity $\Omega=0.32-0.33$, but figures with values of $\Omega$ in the range from $0.31-0.34$ remain compatible with the shape of Kleopatra (rms below $4 \mathrm{~km}$ ), as illustrated in Fig. 5. The $\Omega$ value calculated from Kleopatra's current rotation period $5.385 \mathrm{~h}$ and density $3.38 \pm 0.50 \mathrm{~g} \mathrm{~cm}^{-3}$ is 0.334 , which is in striking agreement with the values reported above. We thus confirm that Kleopatra is an equilibrium shape.

\subsection{Surface acceleration}

To probe the effect of the shape on the internal compaction, we computed the gravitational plus the centrifugal acceleration at each point of the surface and compared it with that of a sphere of equivalent mass (Fig. 6). It appears that the mean acceleration at the surface of Kleopatra amounts to $76 \%$ of that of a sphere of equivalent mass with values below $50 \%$ locally. It follows that Kleopatra's highly elongated shape does not favor compaction and it supports a higher macroprosity compared to more spherical or ellipsoidal bodies of an equivalent mass.

We also computed tangential surface accelerations $\left|a_{\mathrm{t}}\right|$, which indicate possible material motion (Fig. 7). It seems that a convergence is located on the left lobe, at $(x, y, z)=(-120,20,20) \mathrm{km}$, or on the neck $( \pm 20,0,20) \mathrm{km}$. On the other hand, a divergence is on the "hill" at $(50,15,-35) \mathrm{km}$. These locations are common to both ADAM and MPCD models. We do not report on the locations with possible shape artifacts. The maximum accelerations reach $\sim 1 \mathrm{~cm} \mathrm{~s}^{-2}$. Whether this is sufficient to sustain global motion is uncertain because it depends on local topography, regolith structure, roughness, friction, impacts, seismicity, etc. Alternatively, cratering impacts can initiate ballistic transport, with complex near-surface dynamics, determined by the proximity of the critical $\mathrm{L}_{1}$ equipotential.

\subsection{Critical equipotentials}

The effective potential $U_{\text {eff }}=U_{\mathrm{g}}-\frac{1}{2} \omega\left(x^{2}+y^{2}\right)$ was computed using the same algorithm as in Appendix A. We plotted its equipotentials together with the four critical points in Fig. 8. The major result is that the shape extends to a distance that is very close to the critical $\mathrm{L}_{1}$ equipotential. In fact, the ADAM shape model almost touches it at one point in the $(x, y)$ plane. At the same time, they are separated by several (or more) kilometers in the $(x, z)$ plane. An analogous analysis of the MPCD shape shows a very similar result (see Figs. 9 and 10). A minor difference is that the shape touches the equipotential at two different points along its $(x, y)$ and $(x, z)$ circumference. The closest distance is less than a kilometer.

Our work includes three major differences with respect to Hirabayashi \& Scheeres (2014). (i) We used the currently observed $P=5.385 \mathrm{~h}$, together with $\rho=3380 \mathrm{~kg} \mathrm{~m}^{-3}$. Consequently, we did not need any mechanism for a spin-down (e.g., from $2.8 \mathrm{~h}$ ) to explain why the shape is critical. (ii) We did not use any scaling. Instead, the absolute volume is constrained by the $\mathrm{AO}$ observations. (iii) Our $\mathrm{L}_{1}$ point is on the right $(+x)$ and not on the left, implying that the shape model presented here is different to the previous one used by Hirabayashi \& Scheeres (2014). This possibly affects near-surface dynamics and surface locations from which material is more likely to escape.

\section{Discussion}

Our density estimate of $(3.38 \pm 0.50) \mathrm{g} \mathrm{cm}^{-3}$ for Kleopatra is surprising, considering its high radar albedo of $0.43 \pm 0.10$ (Shepard et al. 2018) that implies a high surface bulk density and a large metal content. In comparison, the density of metal-rich asteroid (16) Psyche is $(4.2 \pm 0.6) \mathrm{g} \mathrm{cm}^{-3}$ (Ferrais et al. 2020). One possible explanation for such a low density for Kleopatra is the presence of substantial porosity within the body. Wilson et al. (1999) showed that gravitationally reaccreted asteroids should have porosities of $\sim 20-40 \%$. It is of great interest that the highly elongated shape of Kleopatra actually supports a higher macroporosity than that expected for a spherical or ellipsoidal body. Accordingly, acknowledging a rubble-pile structure of Kleopatra from its specific angular momentum, this range of porosities implies a grain density in the $4.2-5.8 \mathrm{~g} \mathrm{~cm}^{-3}$ range, suggestive of a mixture of $\mathrm{NiFe}$ metal-rich with the inclusion of silicates (Marchis et al. 2003). The presence of silicates is supported by the presence of a 0.9 micron band in Kleopatra's spectrum (Hardersen et al. 2011).

This rubble-pile structure along with its near equilibrium shape is compatible with a formation scenario including a giant impact (Sugiura et al. 2018), followed by reaccumulation during which Kleopatra behaved as a fluid as suggested by Descamps (2010). The dumbbell equilibrium shape also explains the very unusual long neck between the two lobes. The small volume deficit due to the depression observed on lobe B (see Figs. 2 and 4) is coherent with a later smaller impact.

The critical rotation would be $5.250 \mathrm{~h}$ (compared to $5.385 \mathrm{~h}$ ) if we require the $L_{1}$ equipotential to be in contact with the surface. Similarly, the critical density should be $3.2 \mathrm{~g} \mathrm{~cm}^{-3}$. The whole surface actually does not follow the $L_{1}$ equipotential exactly, since even very small impacts could eject fragments beyond the equipotential surface. Moreover, the critical value of $P$ (as well as $\rho$ ) is sensitive to small variations in the topography.

Finally, the low gravity at the edges and along the equator of the body, together with its rubble-pile structure and the equatorial orbits of the moons, opens the possibility that the latter formed via mass shedding. The large offset between the L1 equipotential and the surface at the edges of the asteroid supports this interpretation. 

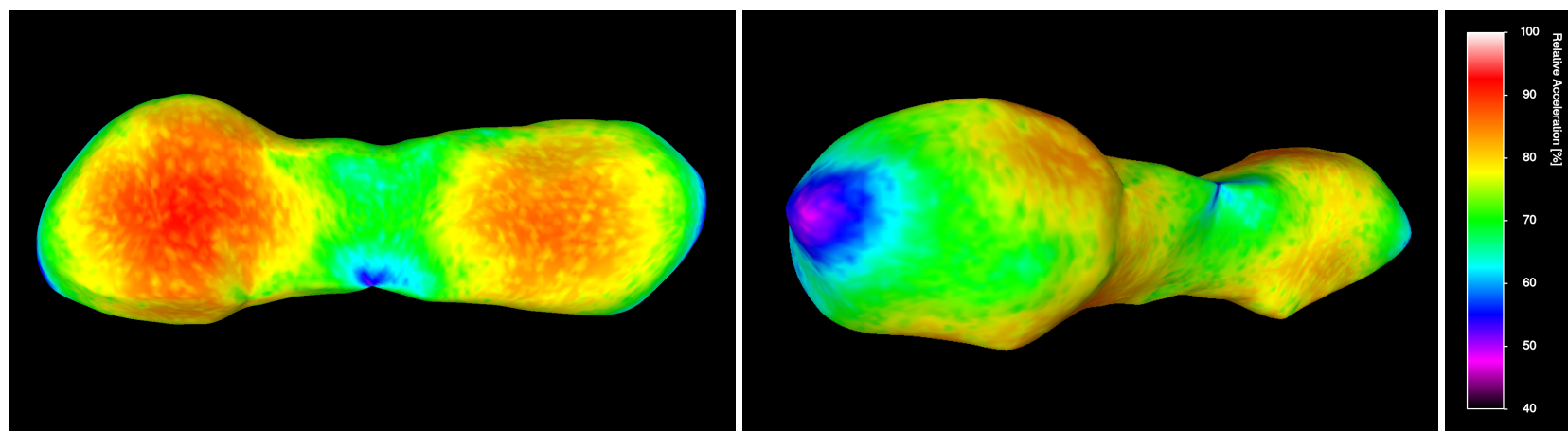

Fig. 6. Local acceleration at the surface of Kleopatra normalized by that of a sphere of an equivalent radius and spin period. View from the $Z+$ (left) axis and oblique view along the equator (right). We note the very low value at the edges and higher values around the two lobes.
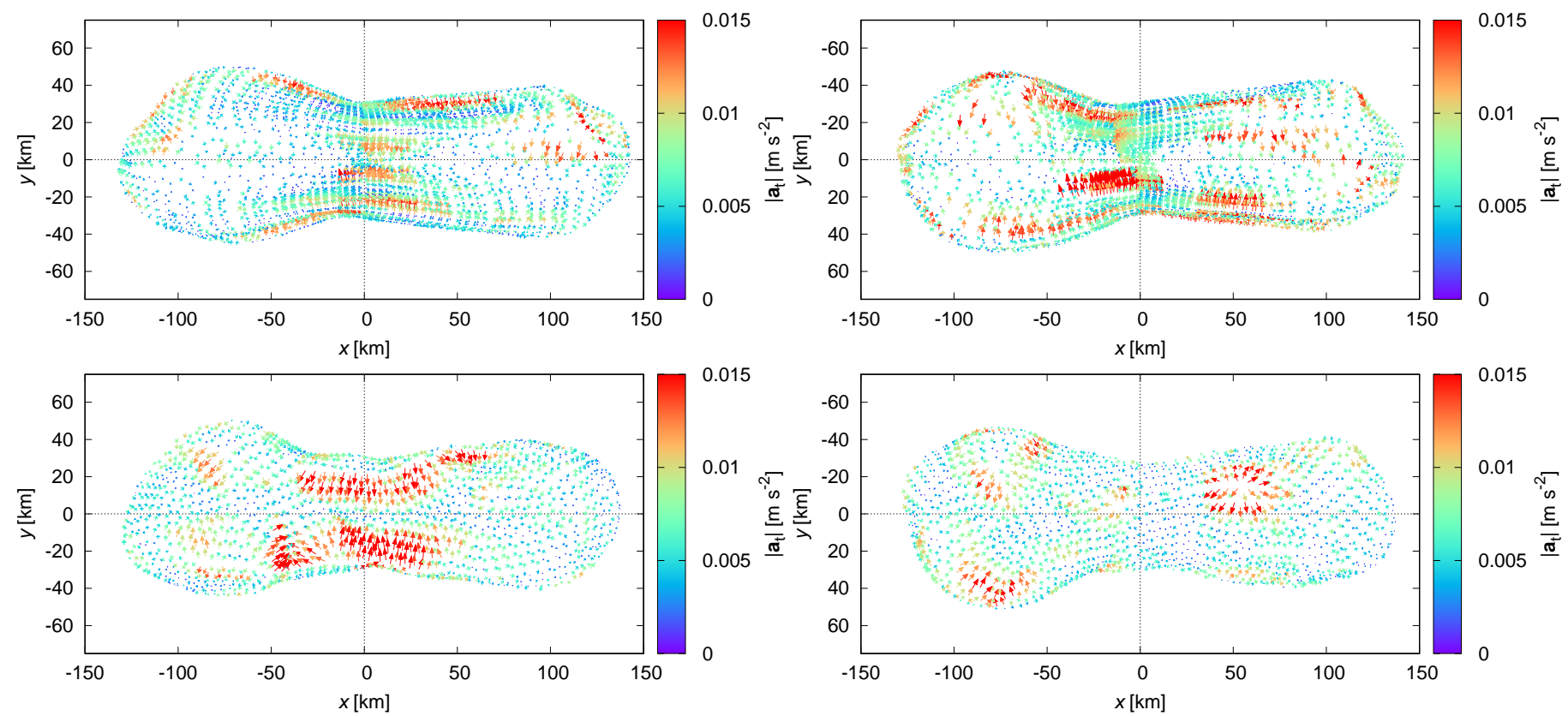

Fig. 7. Tangential surface accelerations $\left|a_{\mathrm{t}}\right|$ computed for ADAM (top) and MPCD (bottom) models. Views on the left are north pole-on (+z), while those on the right are south pole-on $(-z)$.

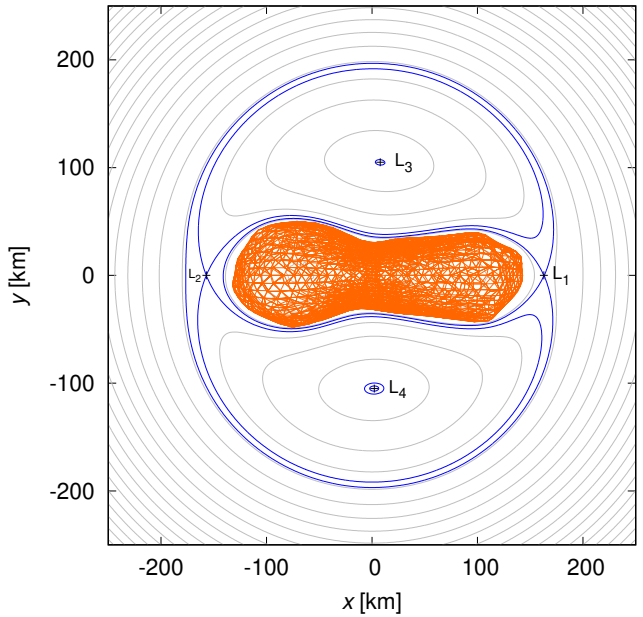

Fig. 8. Effective potential $U_{\text {eff }}$ in the $(x, y)$ plane (gray lines), critical equipotentials (blue lines), and the ADAM shape model (orange). The density is $\rho=3.34 \mathrm{~g} \mathrm{~cm}^{-3}$ and the rotation period $P=5.385 \mathrm{~h}$. Four critical points are denoted: $\mathrm{L}_{1}, \mathrm{~L}_{2}, \mathrm{~L}_{3}$, and $\mathrm{L}_{4}$. The $\mathrm{L}_{1}$ critical point is on the right. The $\mathrm{L}_{1}$ equipotential touches the surface of Kleopatra.

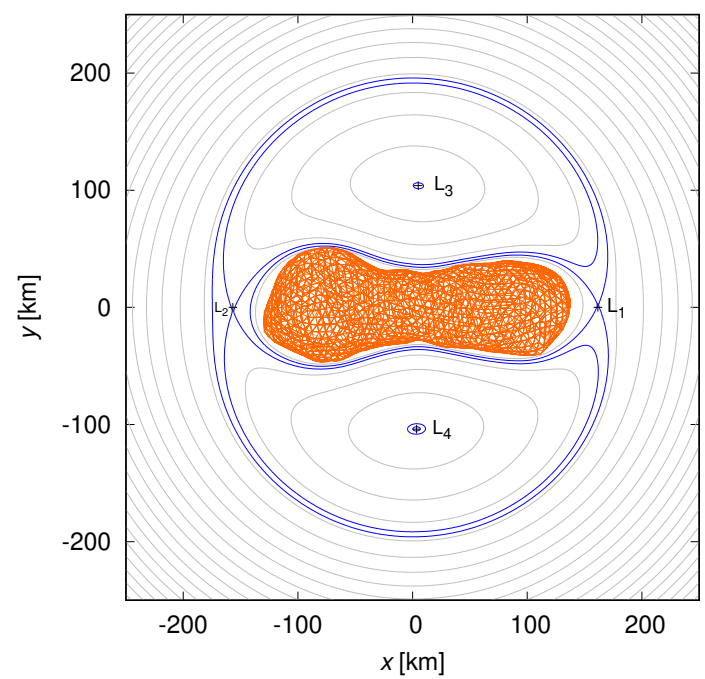

Fig. 9. Effective potential $U_{\text {eff }}$ in the $(x, y)$ plane (gray lines), critical equipotentials (blue lines), for the MPCD model (orange) and density $3.43 \mathrm{~g} \mathrm{~cm}^{-3}$. 


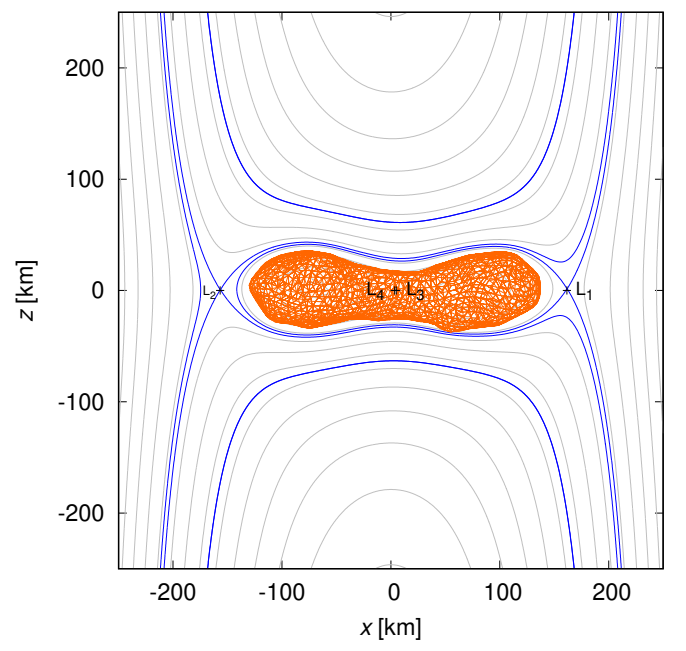

Fig. 10. Effective potential $U_{\text {eff }}$ in the $(x, z)$ plane (gray lines), critical equipotentials (blue lines), for the MPCD model (orange) and density $3.43 \mathrm{~g} \mathrm{~cm}^{-3}$.

\section{Summary and conclusion}

New AO observations of the triple system (216) Kleopatra with VLT/SPHERE allowed us to constrain the 3D shape and mass of the primary with high accuracy (see Brož et al. 2021, companion paper for the mass estimate). Both mass and volume estimates of (216) Kleopatra imply a low density of $(3.38 \pm 0.50) \mathrm{g} \mathrm{cm}^{-3}$. Such low density for a metallic asteroid suggests the presence of substantial porosity within the metal-rich primary or a significant content of silicate in the composition of the asteroid.

This rubble-pile structure along with its near equilibrium shape is compatible with a formation scenario including a giant impact followed by reaccumulation. (216) Kleopatra's rotation period and dumbbell shape imply that it is in a critically rotating state. The low gravity at the edges and along the equator of the body, together with its rubble-pile structure and the equatorial orbits of the moons, opens the possibility that the latter formed via mass shedding as suggested by Descamps et al. (2008).

Future observations of (216) Kleopatra with current AO systems such as SPHERE/ZIMPOL could reveal long-term perturbations in the moon orbits related to the shape of the primary. Similar observations of the primary, but at low phase angles (less than $5 \mathrm{deg}$ ), could also provide more accurate contours and thus help refine its shape.

We can speculate that high resolution images of Kleopatra's surface could help to truly understand the origin of the moons by revealing the presence of surface heterogeneities (e.g., albedo variegations), or anomalies such as concavities, that would help to link the moons directly to their parent body. Future observations with high angular resolution imaging data provided by the next generation of extremely large telescopes could help marginally by providing color and spectroscopic constraints on the moons and refining the shape model of the primary. A future space mission to (216) Kleopatra and its two moons CleoSelene and AlexHelios would definitively shed light on the origin and current dynamics of this complex system. In situ measurements could, for instance, reveal the ejection of particles from Kleopatra similar to what was recently seen on the near-Earth asteroid (101955) Bennu (Lauretta et al. 2019).

Acknowledgements. This material is partially based upon work supported by the National Science Foundation under Grant No. 1743015. This work has been supported by the Czech Science Foundation through grant 20-08218S (J. Hanuš,
J. Durech), 21-11058S (M. Brož) and by the Charles University Research program No. UNCE/SCI/023. P. Vernazza, A. Drouard, M. Ferrais and B. Carry were supported by CNRS/INSU/PNP. M.M. was supported by the National Aeronautics and Space Administration under grant No. 80NSSC18K0849 issued through the Planetary Astronomy Program. The work of TSR was carried out through grant APOSTD/2019/046 by Generalitat Valenciana (Spain). This work was supported by the MINECO (Spanish Ministry of Economy) through grant RTI2018-095076-B-C21 (MINECO/FEDER, UE). The research leading to these results has received funding from the ARC grant for Concerted Research Actions, financed by the Wallonia-Brussels Federation. TRAPPIST is a project funded by the Belgian Fonds (National) de la Recherche Scientifique (F.R.S.-FNRS) under grant FRFC 2.5.594.09.F. TRAPPIST-North is a project funded by the University of Liège, and performed in collaboration with Cadi Ayyad University of Marrakesh. E. Jehin is a FNRS Senior Research Associate. The data presented herein were obtained partially at the W. M. Keck Observatory, which is operated as a scientific partnership among the California Institute of Technology, the University of California and the National Aeronautics and Space Administration. The Observatory was made possible by the generous financial support of the W. M. Keck Foundation. The authors wish to recognize and acknowledge the very significant cultural role and reverence that the summit of Maunakea has always had within the indigenous Hawaiian community. We are most fortunate to have the opportunity to conduct observations from this mountain.

\section{References}

Berthier, J., Vachier, F., Marchis, F., Ďurech, J., \& Carry, B. 2014, Icarus, 239, 118

Beuzit, J. L., Vigan, A., Mouillet, D., et al. 2019, A\&A, 631, A155

Binzel, R. P. 1995, Nature, 374, 767

Brož, M., Marchis, F., Jorda, L. et al. 2021, A\&A, 653, A56

Capanna, C., Jorda, L., Lamy, P. L., \& Gesquiere, G. 2012, AGU Fall Meeting Abs., 2012, P23B

Carlsson, M., \& Lagerkvist, C.-I. 1983, A\&AS, 53, 157

Carry, B., Vachier, F., Berthier, J., et al. 2019, A\&A, 623, A132

Carry, B., Vernazza, P., Vachier, F., et al. 2021, A\&A 650, A129

Cignoni, P., Callieri, M., Corsini, M., et al. 2008, in Eurographics Italian Chapter Conference, eds. V. Scarano, R. D. Chiara, \& U. Erra (The Eurographics Association)

DeMeo, F. E., Binzel, R. P., Slivan, S. M., \& Bus, S. J. 2009, Icarus, 202, 160

Descamps, P. 2010, Icarus, 207, 758

Descamps, P. 2015, Icarus, 245, 64

Descamps, P., Marchis, F., Pollock, J., et al. 2008, Icarus, 196, 578

Descamps, P., Marchis, F., Berthier, J., et al. 2011, Icarus, 211, 1022

Dotto, E., Barucci, M. A., Fulchignoni, M., et al. 1992, A\&AS, 95, 195

Durech, J., Sidorin, V., \& Kaasalainen, M. 2010, A\&A, 513, A46

Fauvaud, S., Sareyan, J. P., Boussuge, J., \& Bernhard, P. 2001, Minor Planet Bull., 28, 44

Ferrais, M., Vernazza, P., Jorda, L., et al. 2020, A\&A, 638, L15

Fétick, R. J., Jorda, L., Vernazza, P., et al. 2019, A\&A, 623, A6

Frank, P., \& Frevert, F. 1988, Sterne und Weltraum, 27, 48

Fusco, T., Mugnier, L. M., Conan, J.-M., et al. 2003, Proc. SPIE, 4839, 1065

Grice, J., Snodgrass, C., Green, S. F., Parley, N. R., \& Carry, B. 2017, in Asteroids, Comets, and Meteors: ACM 2017

Grossman, M., Hoffman, M., \& Duerbeck, H. W. 1981, Minor Planet Bull., 8,14

Hanuš, J., Viikinkoski, M., Marchis, F., et al. 2017, A\&A, 601, A114

Hardersen, P. S., Cloutis, E. A., Reddy, V., Mothé-Diniz, T., \& Emery, J. P. 2011, Meteorit. Planet. Sci., 46, 1910

Harris, A. W., \& Young, J. W. 1989, Icarus, 81, 314

Herald, D., Gault, D., Anderson, R., et al. 2020, MNRAS, 499, 4570

Hirabayashi, M., \& Scheeres, D. J. 2014, ApJ, 780, 160

Hutton, R. G. 1990, Minor Planet Bull., 17, 34

Johnston, W. R. 2021 http://wWw.johnstonsarchive.net/astro/ asteroidmoons.html

Jorda, L., Spjuth, S., Keller, H. U., Lamy, P., \& Llebaria, A. 2010, SPIE Conf. Ser., 7533, 753311

Jorda, L., Gaskell, R., Capanna, C., et al. 2016, Icarus, 277, 257

Kennedy, H. D., \& Tholen, D. J. 1982, Proc. Astron. Soc. Australia, 4, 414

Lauretta, D. S., Hergenrother, C. W., Chesley, S. R., et al. 2019, Science, 366, 3544

Lupishko, D. F., \& Velichko, F. P. 1987, Kinematika i Fizika Nebesnykh Tel, 3, 57

Macintosh, B. A., Anthony, A., Atwood, J., et al. 2014, SPIE Conf. Ser., 9148, 91480J

Marchis, F., Descamps, P., Hestroffer, D., et al. 2003, Icarus, 165, 112 
Marchis, F., Descamps, P., Hestroffer, D., \& Berthier, J. 2005, Nature, 436, 822

Marchis, F., Descamps, P., Berthier, J., et al. 2008, Icarus, 195, 295

Margot, J.-L., Pravec, P., Taylor, P., Carry, B., \& Jacobson, S. 2015, Asteroids IV, eds. P. Michel, F. E. DeMeo, \& W. F. Bottke (Tucson: University of Arizona Press), 355

Merline, W. J., Close, L. M., Dumas, C., et al. 1999, Nature, 401, 565

Ostro, S. J., Hudson, R. S., Nolan, M. C., et al. 2000, Science, 288, 836

Pajuelo, M., Carry, B., Vachier, F., et al. 2018, Icarus, 309, 134

Pilcher, F., \& Tholen, D. J. 1982, Minor Planet Bull., 9, 13

Scaltriti, F., \& Zappala, V. 1978, Icarus, 34, 428

Scheeres, D. J., Britt, D., Carry, B., \& Holsapple, K. A. 2015, Asteroids IV (Tucson: University of Arizona Press)

Shepard, M. K., Timerson, B., Scheeres, D. J., et al. 2018, Icarus, 311, 197

Si, H. 2006, http://wias-berlin.de/software/tetgen/

Sugiura, K., Kobayashi, H., \& Inutsuka, S. 2018, A\&A, 620, A167

Thalmann, C., Schmid, H. M., Boccaletti, A., et al. 2008, Proc. SPIE, 7014, $70143 \mathrm{~F}$

Vernazza, P., Brož, M., Drouard, A., et al. 2018, A\&A, 618, A154

Vernazza, P., Jorda, L., Ševeček, P., et al. 2020, Nat. Astron., 4, 136

Viikinkoski, M., Kaasalainen, M., \& Ďurech, J. 2015a, A\&A, 576, A8

Viikinkoski, M., Kaasalainen, M., Durech, J., et al. 2015b, A\&A, 581, L3

Viikinkoski, M., Vernazza, P., Hanuš, J., et al. 2018, A\&A, 619, L3

Wahhaj, Z., Liu, M. C., Biller, B. A., et al. 2013, ApJ, 779, 80

Warner, B. D. 2006, Minor Planet Bull., 33, 85

Weidenschilling, S. J., Chapman, C. R., Davis, D. R., et al. 1987, Icarus, 70, 191

Weidenschilling, S. J., Chapman, C. R., Davis, D. R., Greenberg, R., \& Levy, D. H. 1990, Icarus, 86, 402

Wilson, L., Keil, K., \& Love, S. J. 1999, Meteorit. Planet. Sci., 34, 479

Yang, B., Wahhaj, Z., Beauvalet, L., et al. 2016, ApJ, 820, L35

Yang, B., Hanuš, J., Carry, B., et al. 2020a, A\&A, 641, A80

Yang, B., Hanuš, J., Brož, M., et al. 2020b, A\&A, 643, A38

Zappala, V., di Martino, M., Scaltriti, F., Djurasevic, G., \& Knezevic, Z. 1983, Icarus, 53, 458

${ }^{1}$ SETI Institute, Carl Sagan Center, 189 Bernado Avenue, Suite 200, Mountain View CA 94043, USA

e-mail: fmarchis@seti.org

2 Aix Marseille Univ, CNRS, LAM, Laboratoire d'Astrophysique de Marseille, Marseille, France
${ }^{3}$ Institute of Astronomy, Faculty of Mathematics and Physics, Charles University, V Holešovičkách 2, 18000 Prague, Czech Republic

${ }^{4}$ IMCCE, Observatoire de Paris, PSL Research University, CNRS, Sorbonne Universités, UPMC Univ Paris 06, Univ. Lille, France

5 Department of Earth, Atmospheric and Planetary Sciences, MIT, 77 Massachusetts Avenue, Cambridge, MA 02139, USA

${ }^{6}$ Mathematics \& Statistics, Tampere University, PO Box 553, 33101 Tampere, Finland

7 Space sciences, Technologies and Astrophysics Research Institute, Université de Liège, Allée du 6 Août 17, 4000 Liège, Belgium

${ }^{8}$ Faculty of Physics, Astronomical Observatory Institute, Adam Mickiewicz University, ul. Słoneczna 36, 60-286 Poznań, Poland

${ }^{9}$ Université Côte d'Azur, Observatoire de la Côte d'Azur, CNRS, Laboratoire Lagrange, France

${ }^{10}$ Observatoire du Bois de Bardon, 16110 Taponnat, France

11 Astronomical Institute of Romanian Academy, 5, Cutitul de Argint Street, 040557 Bucharest, Romania

12 Thirty-Meter-Telescope, 100 West Walnut St, Suite 300, Pasadena, CA 91124, USA

13 Jet Propulsion Laboratory, California Institute of Technology, 4800 Oak Grove Drive, Pasadena, CA 91109, USA

${ }^{14}$ European Space Agency, ESTEC - Scientific Support Office, Keplerlaan 1, Noordwijk 2200 AG, The Netherlands

15 The French Aerospace Lab BP72, 29 avenue de la Division Leclerc, 92322 Chatillon Cedex, France

${ }^{16}$ Open University, School of Physical Sciences, The Open University, MK7 6AA, UK

${ }^{17}$ Laboratoire Atmosphères, Milieux et Observations Spatiales, CNRS \& Université de Versailles Saint-Quentin-en-Yvelines, Guyancourt, France

18 Sección Física, Departamento de Ciencias, Pontificia Universidad Católica del Perú, Apartado 1761, Lima, Peru

${ }^{19}$ Departamento de Fisica, Ingeniería de Sistemas y Teoría de la Señal, Universidad de Alicante, Alicante, Spain

${ }^{20}$ Institut de Ciéncies del Cosmos (ICCUB), Universitat de Barcelona (IEEC-UB), Martí Franqués 1, 08028 Barcelona, Spain

${ }^{21}$ European Southern Observatory (ESO), Alonso de Cordova 3107, 1900 Casilla Vitacura, Santiago, Chile 


\section{Appendix A: Surface slopes}

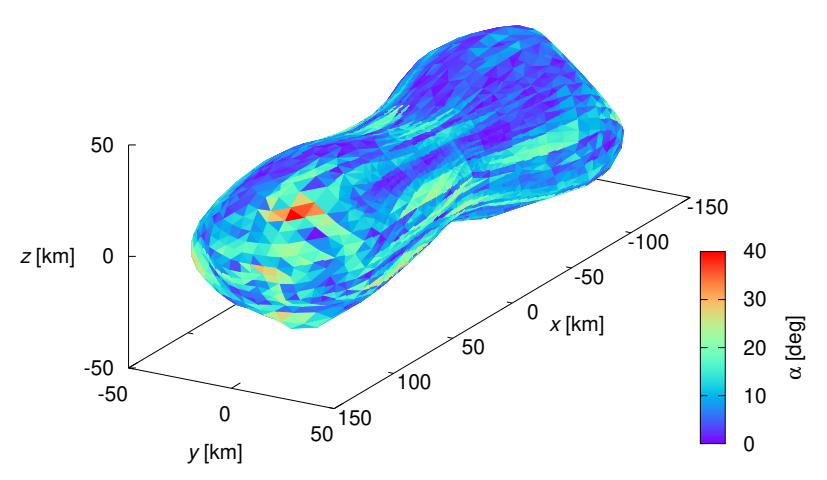

Fig. A.1. Surface slopes $\alpha$ (shown as color) for the ADAM shape model. They were computed for centers of faces and gravitational plus centrifugal accelerations. The density is $\rho=3340 \mathrm{~kg} \mathrm{~m}^{-3}$ and the rotation period $P=5.385 \mathrm{~h}$. Higher slopes are present on the lobes.

Another useful characteristics is the statistics of surface slopes. We computed the slope $\alpha$ as the angle between the local normal and the total acceleration in the corotating frame. To evaluate the respective volumetric integrals, we triangulated the volume via the Tetgen program (Si 2006), with 24099 elements, and the brute-force algorithm. The result is shown in Fig. A.1. Most of the slopes are between $2^{\circ}$ and $15^{\circ}$. Steeper slopes (up to $20^{\circ}$ ) are located on the lobes, at the largest distances from the $z$-axis, and some of them are on the neck. A few outliers (over $25^{\circ}$ ) are related to individual facets, which might be elongated or degenerate.

For comparison, we also show the slopes computed on the basis of the MPCD model (Fig. A.2). This shape model has a lower number of faces which are more regular. Interestingly, the steeper slopes are located elsewhere, mostly on the neck. The slopes on the $+x$ lobe are shallower (around $10^{\circ}$ ). Given the nature of the shape models (ADAM versus MPCD), we think

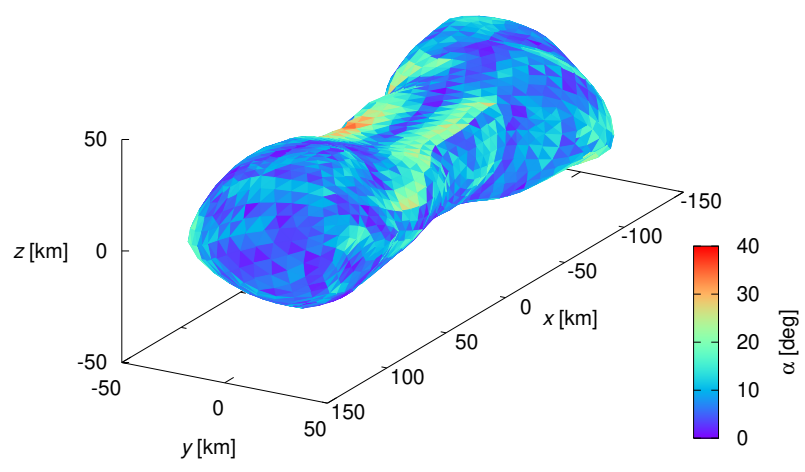

Fig. A.2. Same as Fig. A.1, but for the MPCD model and density $\rho=3430 \mathrm{~kg} \mathrm{~m}^{-3}$. Higher slopes are on the neck.

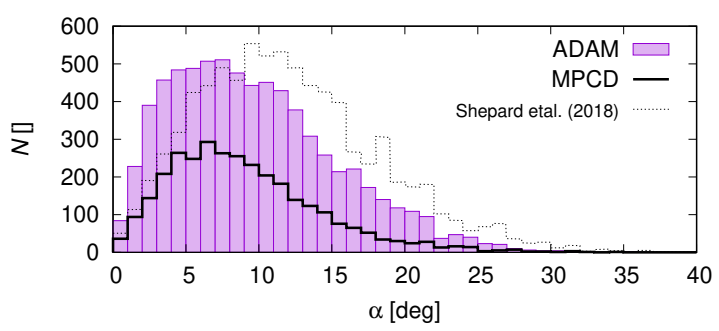

Fig. A.3. Histograms of slopes $\alpha$ for the ADAM and MPCD models. They are most commonly between $2^{\circ}$ and $15^{\circ}$. The distribution is a bit wider for the ADAM model, with a few outliers. For comparison, we also plotted a histogram from Shepard et al. (2018).

that local normals are better constrained by the MPCD model. The overall statistics (Fig. A.3) from both models is comparable, though. A comparison to Shepard et al. (2018) shows that our mean slope is significantly lower $\left(9^{\circ}\right.$ versus $\left.12^{\circ}\right)$. Let us recall, however, that this difference might be partly "enforced" by our/their regularization techniques. 


\section{Appendix B: Additional figures and tables}

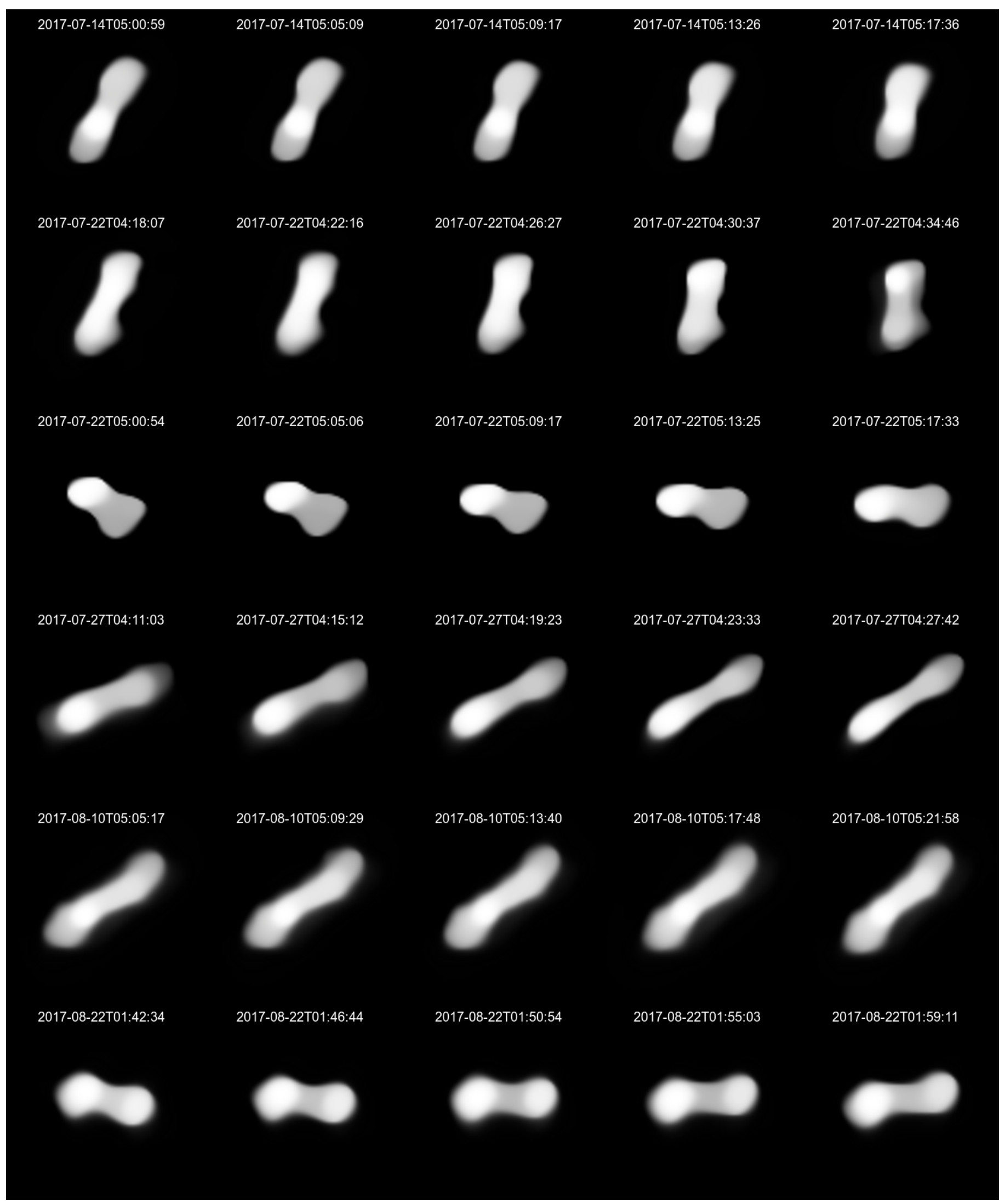

Fig. B.1. Full set of VLT/SPHERE/ZIMPOL images of (216) Kleopatra from the apparition in 2017. The images were deconvolved by the Mistral algorithm. The pixel scale is 3.6 mas. Additional information about the data can be found in Table B.1. 


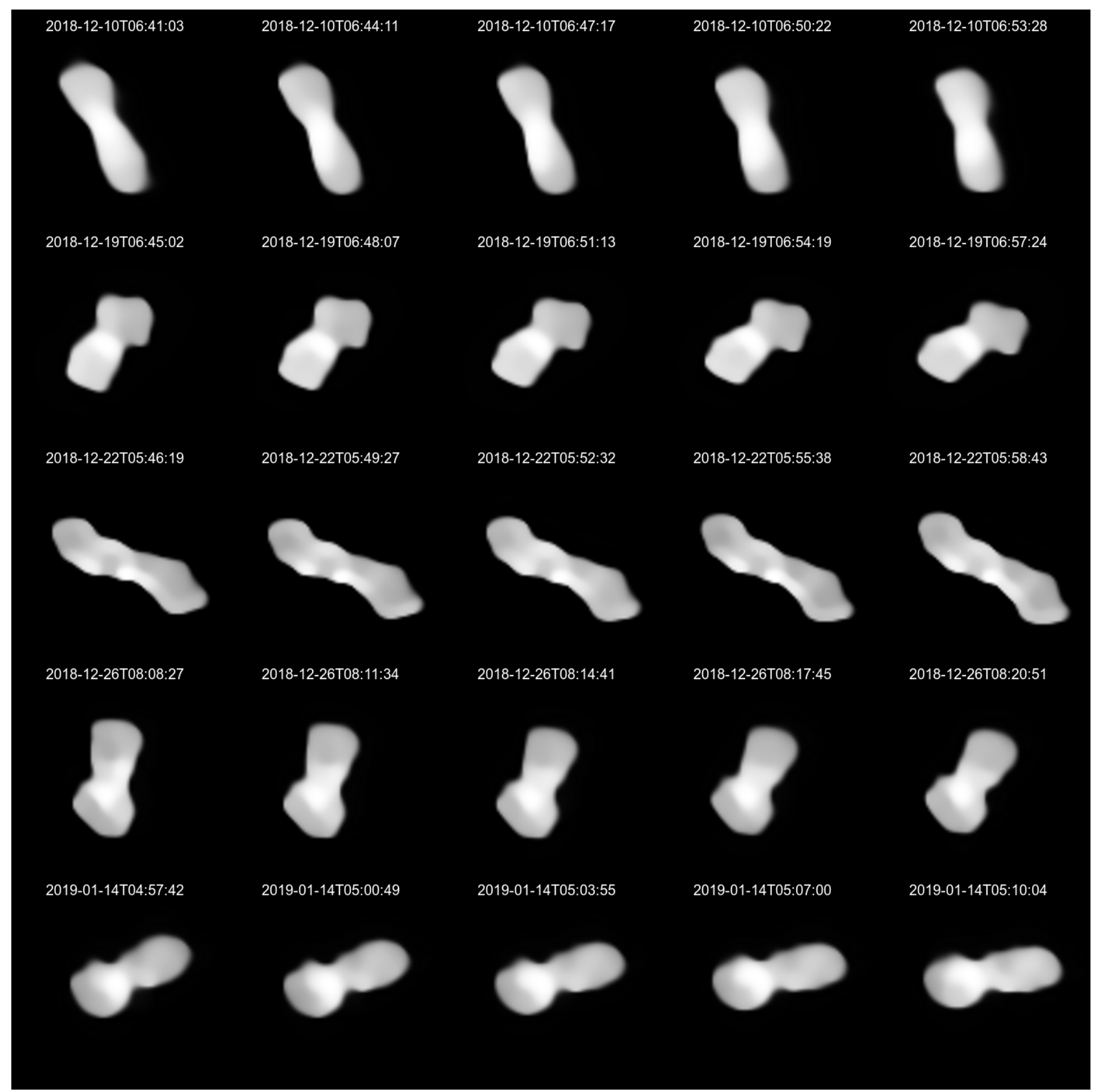

Fig. B.2. Full set of VLT/SPHERE/ZIMPOL images of (216) Kleopatra from the apparition in 2018/2019. The images were deconvolved by the Mistral algorithm. The pixel scale is 3.6 mas. Additional information about the data can be found in Table B.1. 


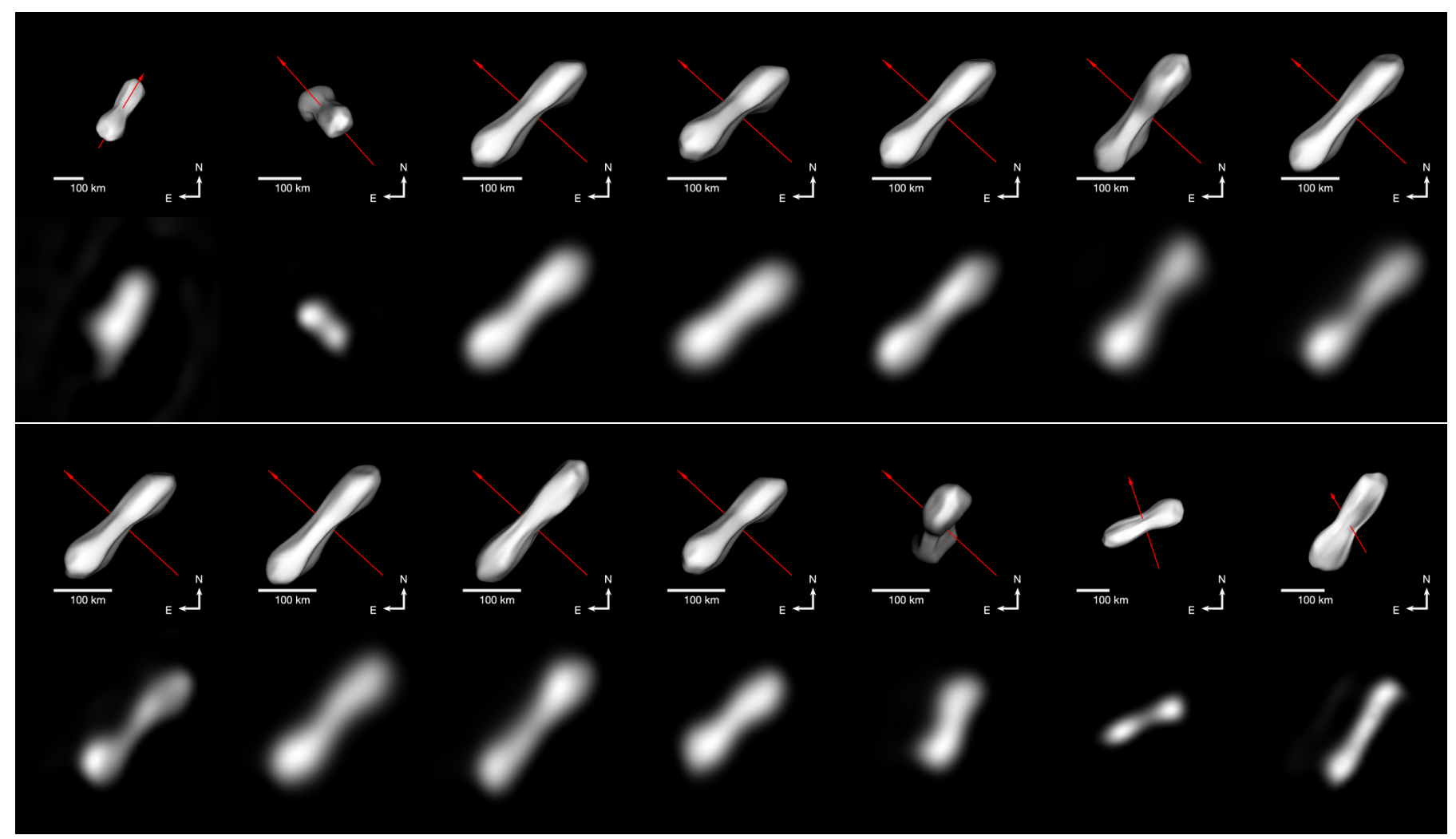

Fig. B.3. Comparison between the disk-resolved images from the Keck/NIRC2 camera and the corresponding projections of our ADAM shape model. The red arrow indicates the orientation of the spin axis. The ordering of the images follows the chronological order in Table B.2. Given the lower resolution of the Keck data (pixel scale 9.942 mas) compared to those of the SPHERE (pixel scale 3.6 mas), the agreement between the images and the model projections is quite reasonable and sufficient. 
A\&A 653, A57 (2021)
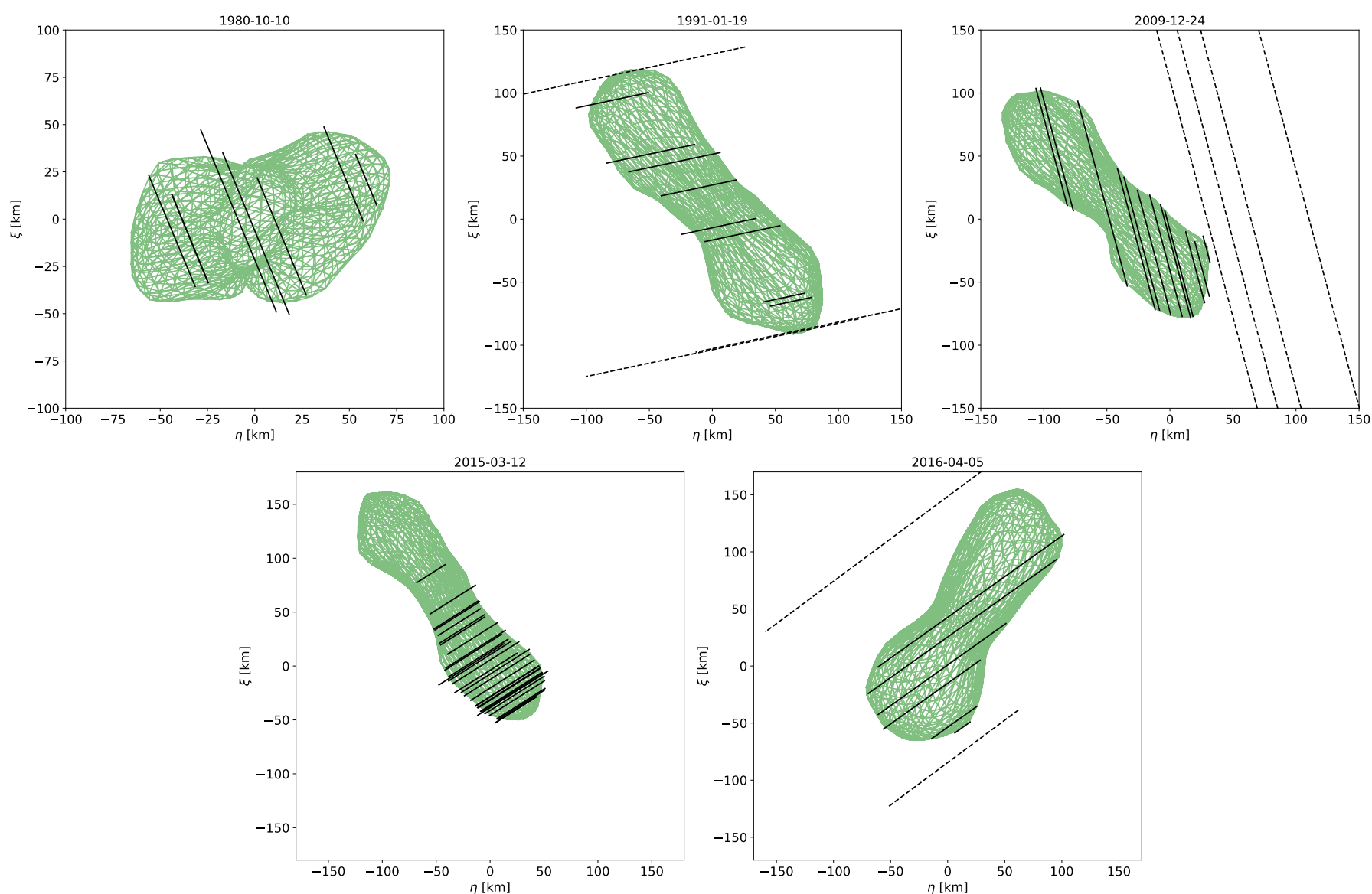

Fig. B.4. Comparison between the stellar occultations of Kleopatra (Herald et al. 2020) and the corresponding projections of our ADAM shape model. Dashed lines are the negative observations. Occultations are ordered chronologically; the first two were not included in the ADAM shape modeling and are shown here for consistency reasons. 
1977-01-15.9

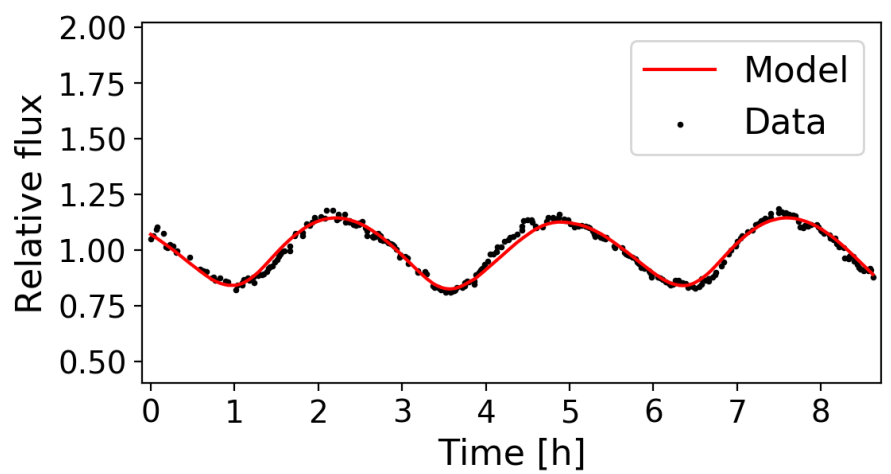

1980-09-30.5

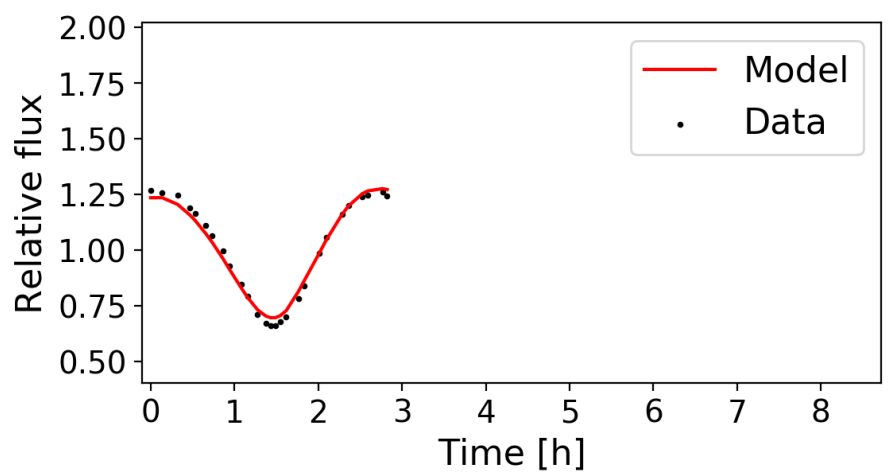

1982-02-13.9

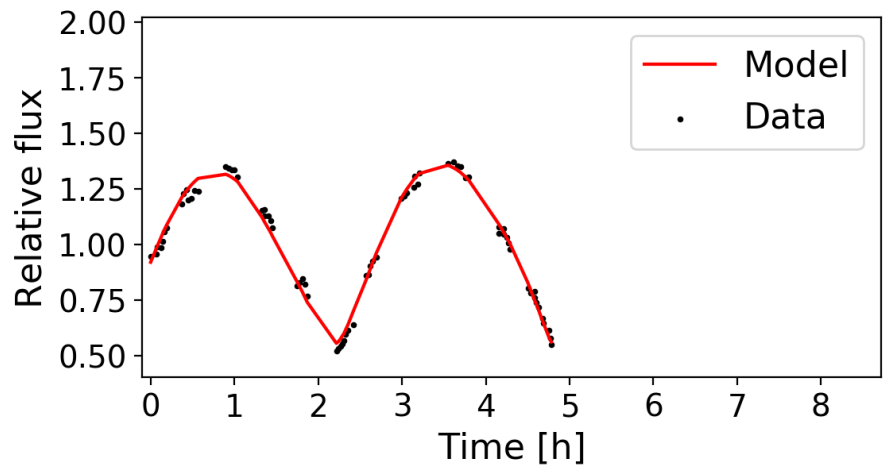

1980-09-15.6

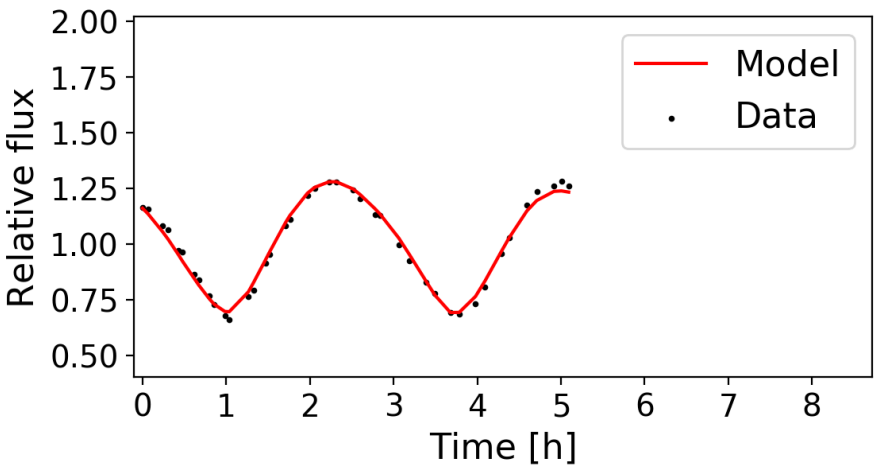

1980-10-01.5

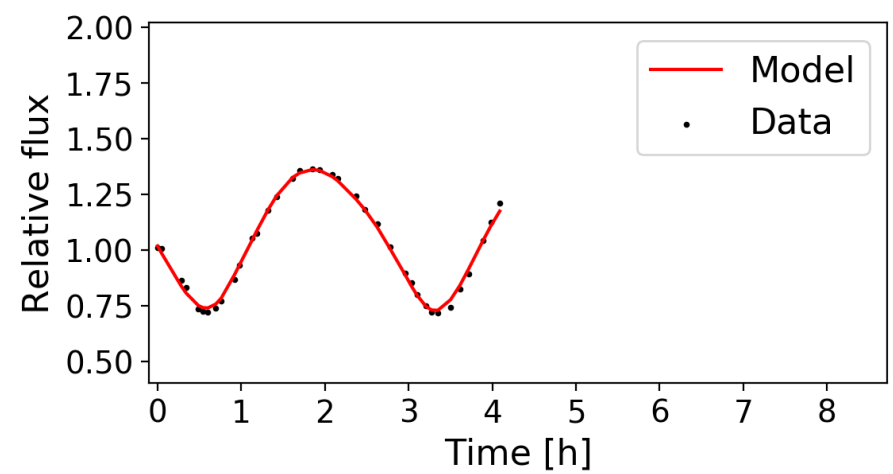

1984-07-05.3

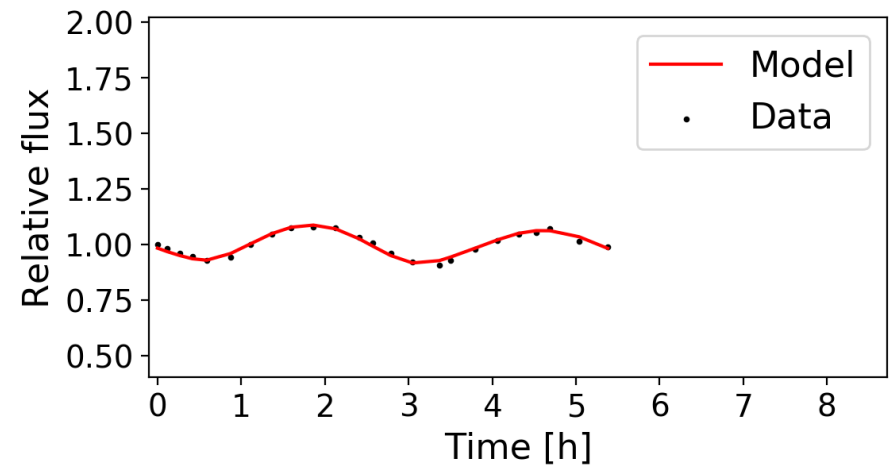

Fig. B.5. Comparison between a subset of optical lightcurves of Kleopatra and the corresponding modeled lightcurves based on our ADAM shape model. The fit to all lightcurves from our dataset is available in the DAMIT database. 
$1985-12-02.9$
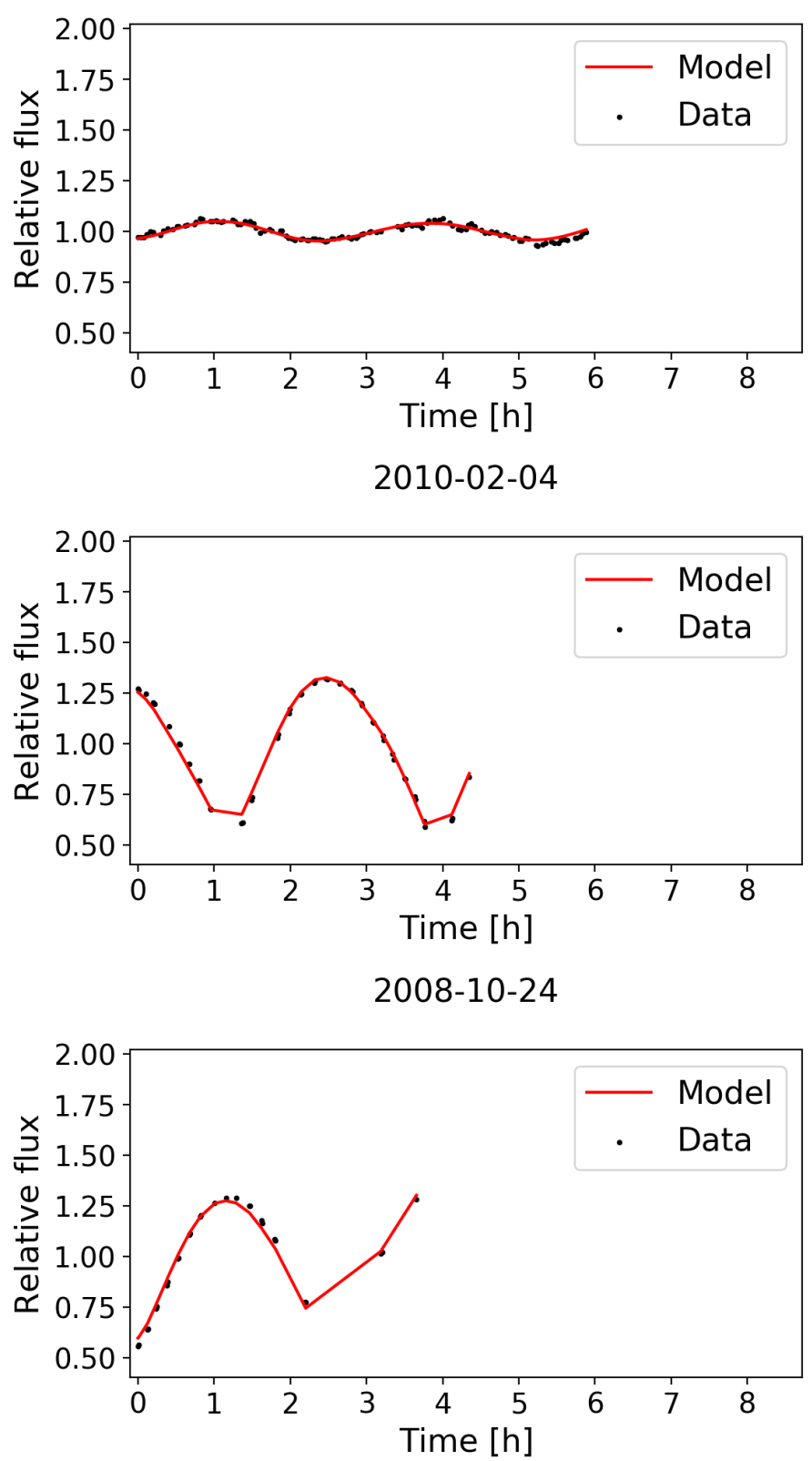

2015-4-15.0

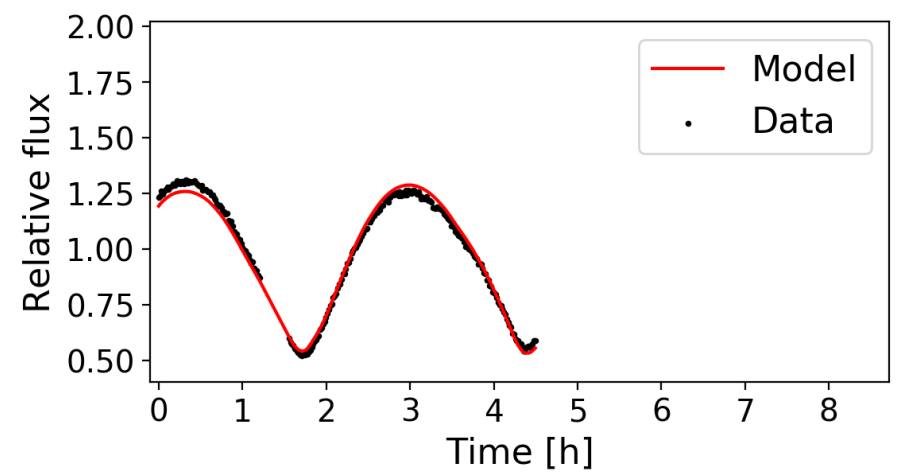

2008-11-12

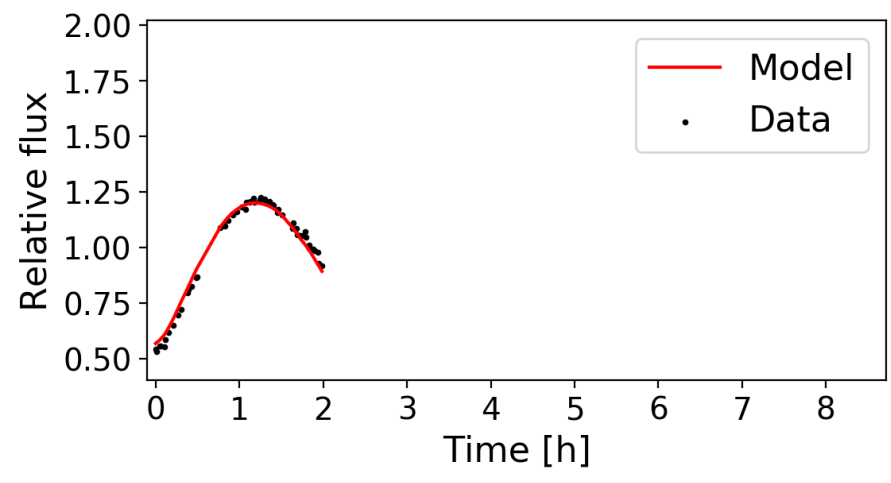

2008-10-28

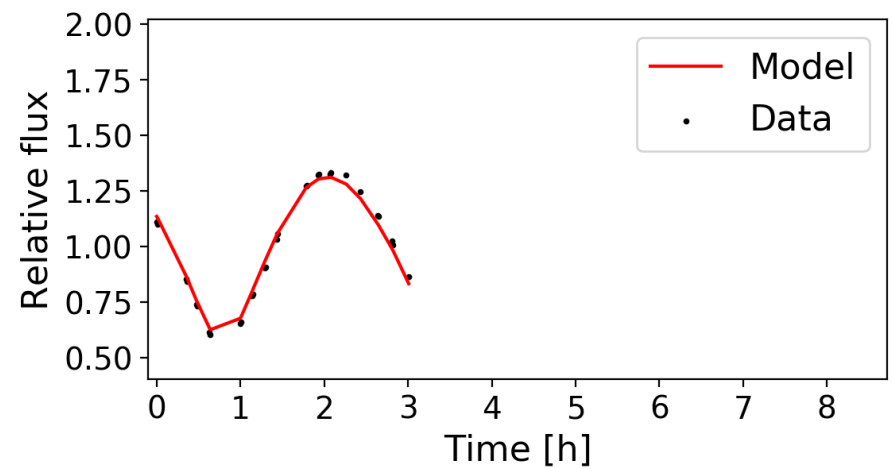

Fig. B.6. Comparison between a subset of optical lightcurves of Kleopatra and the corresponding modeled lightcurves based on our ADAM shape model. The fit to all lightcurves from our dataset is available in the DAMIT database. 
F. Marchis et al.: Critically rotating asteroid (216) Kleopatra
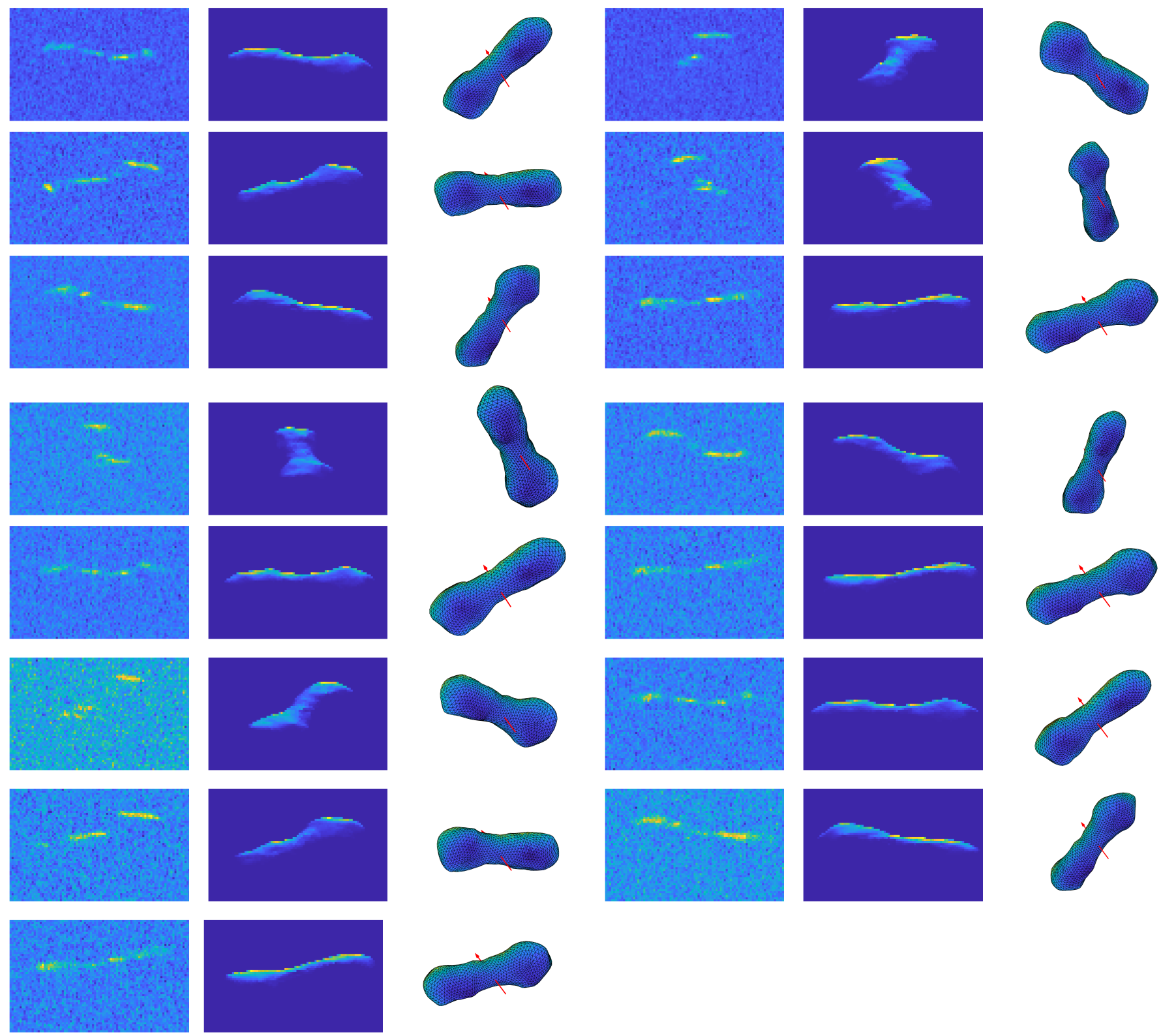

Fig. B.7. Comparison between the delay Doppler images (right) and the synthetic images (middle) based on the best-fitting MPCD shape solution. Three images at slightly different observation times ( $0.6 \%$ of full rotation) overlap to simulate the effect of integration and to improve the contrast. The third column shows the plane of the sky view of the asteroid and the direction of the rotation axis. 
Table B.1. VLT/SPHERE disk-resolved images obtained in the N_R filter by the ZIMPOL camera within the ESO's large program.

\begin{tabular}{|c|c|c|c|c|c|c|c|}
\hline Date & UT & $\begin{array}{c}\operatorname{Exp} \\
\text { (s) }\end{array}$ & Airmass & $\begin{array}{c}\Delta \\
(\mathrm{AU})\end{array}$ & $\begin{array}{c}r \\
(\mathrm{AU})\end{array}$ & $\begin{array}{c}\alpha \\
\left({ }^{\circ}\right)\end{array}$ & $\begin{array}{l}D_{\mathrm{a}} \\
\left({ }^{\prime \prime}\right)\end{array}$ \\
\hline $2017-07-14$ & $5: 00: 59$ & 241 & 1.11 & 1.73 & 2.69 & 8.9 & 0.095 \\
\hline $2017-07-14$ & $5: 05: 09$ & 241 & 1.11 & 1.73 & 2.69 & 8.9 & 0.095 \\
\hline $2017-07-14$ & 5:09:17 & 241 & 1.11 & 1.73 & 2.69 & 8.9 & 0.095 \\
\hline 2017-07-14 & $5: 13: 26$ & 241 & 1.11 & 1.73 & 2.69 & 8.9 & 0.095 \\
\hline 2017-07-14 & $5: 17: 36$ & 241 & 1.11 & 1.73 & 2.69 & 8.9 & 0.095 \\
\hline $2017-07-22$ & 4:18:07 & 241 & 1.11 & 1.69 & 2.66 & 7.9 & 0.097 \\
\hline $2017-07-22$ & $4: 22: 16$ & 241 & 1.11 & 1.69 & 2.66 & 7.9 & 0.097 \\
\hline $2017-07-22$ & $4: 26: 27$ & 241 & 1.11 & 1.69 & 2.66 & 7.9 & 0.097 \\
\hline $2017-07-22$ & $4: 30: 37$ & 241 & 1.11 & 1.69 & 2.66 & 7.9 & 0.097 \\
\hline $2017-07-22$ & $4: 34: 46$ & 241 & 1.11 & 1.69 & 2.66 & 7.9 & 0.097 \\
\hline $2017-07-22$ & $5: 00: 54$ & 241 & 1.11 & 1.69 & 2.66 & 7.9 & 0.097 \\
\hline $2017-07-22$ & $5: 05: 06$ & 241 & 1.11 & 1.69 & 2.66 & 7.9 & 0.097 \\
\hline $2017-07-22$ & $5: 09: 17$ & 241 & 1.12 & 1.69 & 2.66 & 7.9 & 0.097 \\
\hline $2017-07-22$ & $5: 13: 25$ & 241 & 1.12 & 1.69 & 2.66 & 7.9 & 0.097 \\
\hline $2017-07-22$ & $5: 17: 33$ & 241 & 1.12 & 1.69 & 2.66 & 7.9 & 0.097 \\
\hline $2017-07-27$ & $4: 11: 03$ & 241 & 1.11 & 1.68 & 2.65 & 7.9 & 0.098 \\
\hline 2017-07-27 & $4: 15: 12$ & 241 & 1.11 & 1.68 & 2.65 & 7.9 & 0.098 \\
\hline $2017-07-27$ & $4: 19: 23$ & 241 & 1.11 & 1.68 & 2.65 & 7.9 & 0.098 \\
\hline $2017-07-27$ & $4: 23: 33$ & 241 & 1.11 & 1.68 & 2.65 & 7.9 & 0.098 \\
\hline $2017-07-27$ & $4: 27: 42$ & 241 & 1.11 & 1.68 & 2.65 & 7.9 & 0.098 \\
\hline 2017-08-10 & $5: 05: 17$ & 241 & 1.25 & 1.67 & 2.61 & 10.5 & 0.098 \\
\hline 2017-08-10 & $5: 09: 29$ & 241 & 1.26 & 1.67 & 2.61 & 10.5 & 0.098 \\
\hline 2017-08-10 & $5: 13: 40$ & 241 & 1.28 & 1.67 & 2.61 & 10.5 & 0.098 \\
\hline 2017-08-10 & $5: 17: 48$ & 241 & 1.29 & 1.67 & 2.61 & 10.5 & 0.098 \\
\hline 2017-08-10 & $5: 21: 58$ & 241 & 1.31 & 1.67 & 2.61 & 10.5 & 0.098 \\
\hline $2017-08-22$ & $1: 42: 34$ & 241 & 1.10 & 1.71 & 2.58 & 13.9 & 0.096 \\
\hline $2017-08-22$ & $1: 46: 44$ & 241 & 1.10 & 1.71 & 2.58 & 13.9 & 0.096 \\
\hline $2017-08-22$ & $1: 50: 54$ & 241 & 1.10 & 1.71 & 2.58 & 13.9 & 0.096 \\
\hline $2017-08-22$ & $1: 55: 03$ & 241 & 1.09 & 1.71 & 2.58 & 13.9 & 0.096 \\
\hline $2017-08-22$ & 1:59:11 & 241 & 1.09 & 1.71 & 2.58 & 13.9 & 0.096 \\
\hline $2018-12-10$ & $6: 41: 03$ & 176 & 1.13 & 1.54 & 2.37 & 15.9 & 0.107 \\
\hline $2018-12-10$ & $6: 44: 11$ & 176 & 1.13 & 1.54 & 2.37 & 15.9 & 0.107 \\
\hline $2018-12-10$ & $6: 47: 17$ & 176 & 1.13 & 1.54 & 2.37 & 15.9 & 0.107 \\
\hline $2018-12-10$ & $6: 50: 22$ & 176 & 1.13 & 1.54 & 2.37 & 15.9 & 0.107 \\
\hline $2018-12-10$ & $6: 53: 28$ & 176 & 1.13 & 1.54 & 2.37 & 15.9 & 0.107 \\
\hline $2018-12-19$ & $6: 45: 02$ & 176 & 1.12 & 1.51 & 2.40 & 12.9 & 0.109 \\
\hline 2018-12-19 & $6: 48: 07$ & 176 & 1.13 & 1.51 & 2.40 & 12.9 & 0.109 \\
\hline 2018-12-19 & $6: 51: 13$ & 176 & 1.13 & 1.51 & 2.40 & 12.9 & 0.109 \\
\hline $2018-12-19$ & $6: 54: 19$ & 176 & 1.13 & 1.51 & 2.40 & 12.9 & 0.109 \\
\hline 2018-12-19 & $6: 57: 24$ & 176 & 1.13 & 1.51 & 2.40 & 12.9 & 0.109 \\
\hline $2018-12-22$ & $5: 46: 19$ & 176 & 1.12 & 1.50 & 2.40 & 12.0 & 0.109 \\
\hline $2018-12-22$ & $5: 49: 27$ & 176 & 1.12 & 1.50 & 2.40 & 12.0 & 0.109 \\
\hline $2018-12-22$ & $5: 52: 32$ & 176 & 1.12 & 1.50 & 2.40 & 12.0 & 0.109 \\
\hline $2018-12-22$ & $5: 55: 38$ & 176 & 1.12 & 1.50 & 2.40 & 12.0 & 0.109 \\
\hline $2018-12-22$ & $5: 58: 43$ & 176 & 1.12 & 1.50 & 2.40 & 12.0 & 0.109 \\
\hline $2018-12-26$ & $8: 08: 27$ & 176 & 1.37 & 1.50 & 2.41 & 10.7 & 0.109 \\
\hline $2018-12-26$ & $8: 11: 34$ & 176 & 1.38 & 1.50 & 2.41 & 10.7 & 0.109 \\
\hline $2018-12-26$ & $8: 14: 41$ & 176 & 1.40 & 1.50 & 2.41 & 10.7 & 0.109 \\
\hline $2018-12-26$ & $8: 17: 45$ & 176 & 1.41 & 1.50 & 2.41 & 10.7 & 0.109 \\
\hline $2018-12-26$ & $8: 20: 51$ & 176 & 1.43 & 1.50 & 2.41 & 10.7 & 0.109 \\
\hline 2019-01-14 & $4: 57: 42$ & 176 & 1.13 & 1.52 & 2.46 & 8.4 & 0.108 \\
\hline 2019-01-14 & $5: 00: 49$ & 176 & 1.14 & 1.52 & 2.46 & 8.4 & 0.108 \\
\hline 2019-01-14 & $5: 03: 55$ & 176 & 1.14 & 1.52 & 2.46 & 8.4 & 0.108 \\
\hline 2019-01-14 & $5: 07: 00$ & 176 & 1.14 & 1.52 & 2.46 & 8.4 & 0.108 \\
\hline 2019-01-14 & $5: 10: 04$ & 176 & 1.15 & 1.52 & 2.46 & 8.4 & 0.108 \\
\hline
\end{tabular}

Notes. For each observation, we provide the epoch, the exposure time, the airmass, the distance to the Earth $\Delta$ and the Sun $r$, the phase angle $\alpha$, and the angular diameter $D_{\mathrm{a}}$ of Kleopatra. $241 \mathrm{~s}(176 \mathrm{~s})$ total exposure time corresponds to $1.24 \%(0.91 \%)$ of the rotation period. 
Table B.2. Disk-resolved images obtained by the NIRC2 camera mounted on the Keck II telescope.

\begin{tabular}{|c|c|c|c|c|c|c|c|c|c|}
\hline Date & UT & Filter & $\begin{array}{c}\text { Exp } \\
\text { (s) }\end{array}$ & Airmass & $\begin{array}{c}\Delta \\
(\mathrm{AU}) \\
\end{array}$ & $\begin{array}{c}r \\
(\mathrm{AU}) \\
\end{array}$ & $\begin{array}{c}\alpha \\
\left({ }^{\circ}\right) \\
\end{array}$ & $\begin{array}{l}D_{\mathrm{a}} \\
\left({ }^{\prime \prime}\right)\end{array}$ & Program PI \\
\hline 2002-05-07 & $10: 54: 36$ & $H$ & 60 & 1.20 & 2.45 & 3.46 & 1.8 & 0.067 & J.L. Margot \\
\hline 2003-07-14 & $11: 11: 35$ & $K p$ & 10 & 1.05 & 1.70 & 2.65 & 9.8 & 0.097 & W. Merline \\
\hline 2008-09-19 & $06: 16: 59$ & PK50_1.5 & 60 & 1.47 & 1.24 & 2.22 & 7.5 & 0.132 & F. Marchis \\
\hline 2008-09-19 & $06: 28: 57$ & PK50_1.5 & 60 & 1.40 & 1.24 & 2.22 & 7.5 & 0.132 & F. Marchis \\
\hline 2008-09-19 & $11: 38: 20$ & PK50_1.5 & 60 & 1.18 & 1.24 & 2.22 & 7.5 & 0.132 & F. Marchis \\
\hline 2008-10-05 & 09:12:57 & PK50_1.5 & 60 & 1.05 & 1.26 & 2.19 & 12.3 & 0.130 & F. Marchis \\
\hline 2008-10-05 & 09:48:54 & PK50_1.5 & 60 & 1.10 & 1.26 & 2.19 & 12.3 & 0.130 & F. Marchis \\
\hline 2008-10-05 & 10:03:40 & $H$ & 30 & 1.13 & 1.26 & 2.19 & 12.3 & 0.130 & F. Marchis \\
\hline 2008-10-06 & $07: 18: 06$ & PK50_1.5 & 60 & 1.05 & 1.26 & 2.19 & 12.6 & 0.130 & F. Marchis \\
\hline 2008-10-06 & 09:49:50 & PK50_1.5 & 60 & 1.11 & 1.26 & 2.19 & 12.7 & 0.130 & F. Marchis \\
\hline 2008-10-09 & $05: 45: 41$ & PK50_1.5 & 60 & 1.23 & 1.27 & 2.18 & 13.8 & 0.129 & F. Marchis \\
\hline 2008-10-09 & $09: 35: 21$ & PK50_1.5 & 60 & 1.11 & 1.27 & 2.18 & 13.8 & 0.129 & F. Marchis \\
\hline 2012-08-10 & $06: 14: 05$ & $K p$ & 0.8 & 1.14 & 2.23 & 2.92 & 16.6 & 0.074 & W. Merline \\
\hline 2013-08-26 & $15: 38: 25$ & $K p$ & 0.2 & 1.00 & 1.69 & 2.11 & 28.2 & 0.097 & W. Merline \\
\hline
\end{tabular}

Notes. For each observation, we provide the epoch, the filter, the exposure time, the airmass, the distance to the Earth $\Delta$ and the Sun $r$, the phase angle $\alpha$, the angular diameter $D_{\mathrm{a}}$ of Kleopatra and the program PI.

Table B.3. Optical disk-integrated lightcurves of Kleopatra used for ADAM shape modeling.

\begin{tabular}{|c|c|c|c|c|c|c|c|}
\hline $\mathrm{N}$ & Epoch & $N_{\mathrm{p}}$ & $\begin{array}{c}\Delta \\
(\mathrm{AU})\end{array}$ & $\begin{array}{c}r \\
(\mathrm{AU})\end{array}$ & $\begin{array}{l}\varphi \\
\left({ }^{\circ}\right)\end{array}$ & Filter & Reference \\
\hline 1 & $1977-01-15.9$ & 277 & 1.57 & 2.51 & 8.1 & $V$ & Scaltriti \& Zappala (1978) \\
\hline 2 & 1977-01-16.9 & 245 & 1.57 & 2.51 & 8.1 & $V$ & Scaltriti \& Zappala (1978) \\
\hline 3 & $1980-09-06.3$ & 23 & 1.24 & 2.20 & 10.3 & $C$ & Pilcher \& Tholen (1982) \\
\hline 4 & 1980-09-07.3 & 27 & 1.23 & 2.20 & 9.9 & $C$ & Pilcher \& Tholen (1982) \\
\hline 5 & 1980-09-09.3 & 20 & 1.23 & 2.20 & 9.2 & $C$ & Pilcher \& Tholen (1982) \\
\hline 6 & 1980-09-11.1 & 11 & 1.22 & 2.19 & 8.6 & $C$ & Pilcher \& Tholen (1982) \\
\hline 7 & $1980-09-14.3$ & 25 & 1.21 & 2.19 & 7.8 & $C$ & Pilcher \& Tholen (1982) \\
\hline 8 & 1980-09-15.6 & 41 & 1.20 & 2.19 & 7.5 & $V$ & Kennedy \& Tholen (1982) \\
\hline 9 & 1980-09-18.2 & 18 & 1.20 & 2.18 & 7.1 & $C$ & Pilcher \& Tholen (1982) \\
\hline 10 & $1980-09-20.3$ & 14 & 1.19 & 2.18 & 7.0 & $V$ & Harris \& Young (1989) \\
\hline 11 & 1980-09-20.3 & 18 & 1.19 & 2.18 & 7.0 & $C$ & Pilcher \& Tholen (1982) \\
\hline 12 & 1980-09-21.3 & 10 & 1.19 & 2.18 & 7.0 & $C$ & Pilcher \& Tholen (1982) \\
\hline 13 & 1980-09-27.1 & 11 & 1.19 & 2.17 & 7.7 & $C$ & Pilcher \& Tholen (1982) \\
\hline 14 & $1980-09-29.2$ & 21 & 1.19 & 2.16 & 8.2 & $C$ & Pilcher \& Tholen (1982) \\
\hline 15 & 1980-09-30.5 & 27 & 1.19 & 2.16 & 8.6 & $V$ & Kennedy \& Tholen (1982) \\
\hline 16 & $1980-10-01.5$ & 37 & 1.19 & 2.16 & 8.9 & $V$ & Kennedy \& Tholen (1982) \\
\hline 17 & $1980-10-02.0$ & 63 & 1.19 & 2.16 & 9.0 & $V$ & Zappala et al. (1983) \\
\hline 18 & $1980-10-02.1$ & 44 & 1.19 & 2.16 & 9.1 & $V$ & Zappala et al. (1983) \\
\hline 19 & $1980-10-02.6$ & 9 & 1.19 & 2.16 & 9.3 & $V$ & Kennedy \& Tholen (1982) \\
\hline 20 & $1980-10-06.2$ & 37 & 1.20 & 2.15 & 10.6 & $C$ & Pilcher \& Tholen (1982) \\
\hline 21 & 1980-10-09.2 & 30 & 1.21 & 2.15 & 11.7 & $C$ & Pilcher \& Tholen (1982) \\
\hline 22 & $1980-11-06.2$ & 5 & 1.36 & 2.11 & 22.0 & $V$ & Harris \& Young (1989) \\
\hline 23 & $1980-11-07.8$ & 199 & 1.38 & 2.11 & 22.4 & $V$ & Grossman et al. (1981) \\
\hline 24 & $1980-11-10.2$ & 24 & 1.40 & 2.11 & 23.0 & $C$ & Pilcher \& Tholen (1982) \\
\hline 25 & $1981-12-01.2$ & 19 & 2.48 & 2.72 & 21.2 & $V$ & Weidenschilling et al. (1987) \\
\hline 26 & $1981-12-02.4$ & 17 & 2.47 & 2.72 & 21.2 & $V$ & Weidenschilling et al. (1987) \\
\hline 27 & 1982-02-13.9 & 74 & 1.96 & 2.91 & 6.4 & $V$ & Zappala et al. (1983) \\
\hline 28 & $1982-02-14.0$ & 36 & 1.96 & 2.91 & 6.4 & $V$ & Zappala et al. (1983) \\
\hline 29 & $1982-02-20.3$ & 30 & 1.97 & 2.93 & 6.0 & $V$ & Weidenschilling et al. (1987) \\
\hline 30 & $1982-03-23.2$ & 162 & 2.18 & 3.00 & 12.6 & $V$ & Carlsson \& Lagerkvist (1983) \\
\hline 31 & 1982-03-26.9 & 126 & 2.22 & 3.01 & 13.5 & $V$ & Zappala et al. (1983) \\
\hline 32 & $1983-02-20.3$ & 18 & 3.01 & 3.48 & 15.4 & $V$ & Weidenschilling et al. (1987) \\
\hline 33 & $1983-02-21.3$ & 8 & 2.99 & 3.48 & 15.3 & $V$ & Weidenschilling et al. (1987) \\
\hline 34 & $1983-02-22.4$ & 5 & 2.98 & 3.48 & 15.2 & $V$ & Weidenschilling et al. (1987) \\
\hline
\end{tabular}

Notes. For each lightcurve, the table gives the epoch, the number of individual measurements $N_{\mathrm{p}}$, asteroid's distances to the Earth $\Delta$ and the Sun $r$, phase angle $\varphi$, photometric filter and reference. 
Table B.3. continued.

\begin{tabular}{|c|c|c|c|c|c|c|c|}
\hline $\mathrm{N}$ & Epoch & $N_{\mathrm{p}}$ & $\begin{array}{c}\Delta \\
(\mathrm{AU})\end{array}$ & $\begin{array}{c}r \\
(\mathrm{AU}) \\
\end{array}$ & $\begin{array}{l}\varphi \\
\left({ }^{\circ}\right)\end{array}$ & Filter & Reference \\
\hline 35 & $1983-03-28.3$ & 26 & 2.60 & 3.49 & 8.5 & $V$ & Weidenschilling et al. (1987) \\
\hline 36 & $1983-03-29.3$ & 12 & 2.59 & 3.49 & 8.2 & $V$ & Weidenschilling et al. (1987) \\
\hline 37 & $1983-05-21.3$ & 15 & 2.60 & 3.50 & 8.8 & $V$ & Weidenschilling et al. (1987) \\
\hline 38 & $1983-05-22.3$ & 18 & 2.61 & 3.50 & 9.1 & $V$ & Weidenschilling et al. (1987) \\
\hline 39 & $1983-05-23.2$ & 6 & 2.61 & 3.50 & 9.4 & $V$ & Weidenschilling et al. (1987) \\
\hline 40 & 1984-05-08.4 & 10 & 2.40 & 3.10 & 15.3 & $V$ & Weidenschilling et al. (1987) \\
\hline 41 & $1984-05-09.4$ & 18 & 2.39 & 3.10 & 15.2 & $V$ & Weidenschilling et al. (1987) \\
\hline 42 & $1984-05-10.2$ & 7 & 2.38 & 3.10 & 15.0 & $V$ & Weidenschilling et al. (1987) \\
\hline 43 & $1984-07-05.3$ & 24 & 2.00 & 2.97 & 6.9 & $V$ & Weidenschilling et al. (1987) \\
\hline 44 & $1985-10-21.3$ & 35 & 1.25 & 2.11 & 17.5 & $V$ & Weidenschilling et al. (1987) \\
\hline 45 & 1985-11-09.9 & 63 & 1.17 & 2.13 & 8.8 & $V$ & Lupishko \& Velichko (1987) \\
\hline 46 & $1985-12-02.9$ & 117 & 1.19 & 2.16 & 7.3 & $V$ & Dotto et al. (1992) \\
\hline 47 & $1985-12-08.0$ & 84 & 1.22 & 2.16 & 9.4 & $V$ & Dotto et al. (1992) \\
\hline 48 & $1986-01-17.2$ & 34 & 1.58 & 2.23 & 22.7 & $V$ & Weidenschilling et al. (1987) \\
\hline 49 & 1987-02-03.4 & 33 & 2.41 & 3.15 & 13.6 & $V$ & Weidenschilling et al. (1990) \\
\hline 50 & 1987-03-06.0 & 35 & 2.25 & 3.21 & 5.2 & $C$ & Frank \& Frevert (1988) \\
\hline 51 & $1988-04-23.4$ & 18 & 2.51 & 3.46 & 6.0 & $V$ & Weidenschilling et al. (1990) \\
\hline 52 & $1988-04-25.2$ & 21 & 2.49 & 3.46 & 5.5 & $V$ & Weidenschilling et al. (1990) \\
\hline 53 & $1989-07-05.2$ & 48 & 1.77 & 2.68 & 12.1 & $V B$ & Hutton (1990) \\
\hline 54 & 1994-09-05.1 & 16 & 1.26 & 2.23 & 9.7 & $R$ & Fauvaud et al. (2001) \\
\hline 55 & 1994-09-06.1 & 15 & 1.25 & 2.22 & 9.4 & $R$ & Fauvaud et al. (2001) \\
\hline 56 & 1994-09-07.2 & 50 & 1.25 & 2.22 & 9.1 & $R$ & Fauvaud et al. (2001) \\
\hline 57 & 1994-09-08.1 & 163 & 1.25 & 2.22 & 8.8 & $R$ & Fauvaud et al. (2001) \\
\hline 58 & 2006-04-02.3 & 134 & 2.42 & 3.42 & 2.1 & $R$ & Warner (2006) \\
\hline 59 & 2006-04-03.2 & 149 & 2.42 & 3.42 & 2.1 & $R$ & Warner (2006) \\
\hline 60 & $2008-07-26$ & 56 & 1.59 & 2.33 & 21.0 & $C$ & Grice et al. (2017) \\
\hline 61 & $2008-07-27$ & 61 & 1.58 & 2.33 & 20.8 & $C$ & Grice et al. (2017) \\
\hline 62 & $2008-07-28$ & 65 & 1.57 & 2.32 & 20.6 & $C$ & Grice et al. (2017) \\
\hline 63 & 2008-07-29 & 53 & 1.56 & 2.32 & 20.4 & $C$ & Grice et al. (2017) \\
\hline 64 & $2008-07-30$ & 61 & 1.55 & 2.32 & 20.1 & $C$ & Grice et al. (2017) \\
\hline 65 & 2008-08-01 & 83 & 1.53 & 2.31 & 19.7 & $C$ & Grice et al. (2017) \\
\hline 66 & 2008-08-02 & 37 & 1.52 & 2.31 & 19.4 & $C$ & Grice et al. (2017) \\
\hline 67 & 2008-08-04 & 41 & 1.50 & 2.31 & 18.9 & $C$ & Grice et al. (2017) \\
\hline 68 & 2008-08-05 & 49 & 1.49 & 2.31 & 18.7 & $C$ & Grice et al. (2017) \\
\hline 69 & 2008-08-06 & 59 & 1.48 & 2.30 & 18.4 & $C$ & Grice et al. (2017) \\
\hline 70 & 2008-08-07 & 38 & 1.47 & 2.30 & 18.1 & $C$ & Grice et al. (2017) \\
\hline 71 & 2008-08-09 & 69 & 1.45 & 2.30 & 17.6 & $C$ & Grice et al. (2017) \\
\hline 72 & 2008-08-10 & 61 & 1.44 & 2.30 & 17.3 & $C$ & Grice et al. (2017) \\
\hline 73 & 2008-08-11 & 65 & 1.43 & 2.29 & 17.0 & $C$ & Grice et al. (2017) \\
\hline 74 & $2008-09-23$ & 39 & 1.24 & 2.21 & 8.4 & $C$ & Grice et al. (2017) \\
\hline 75 & $2008-09-23$ & 40 & 1.24 & 2.21 & 8.4 & $C$ & Grice et al. (2017) \\
\hline 76 & $2008-10-03$ & 67 & 1.25 & 2.19 & 11.7 & $C$ & Grice et al. (2017) \\
\hline 77 & 2008-10-04 & 70 & 1.25 & 2.19 & 12.1 & $C$ & Grice et al. (2017) \\
\hline 78 & $2008-10-05$ & 37 & 1.26 & 2.19 & 12.5 & $C$ & Grice et al. (2017) \\
\hline 79 & 2008-10-06 & 48 & 1.26 & 2.19 & 12.9 & $C$ & Grice et al. (2017) \\
\hline 80 & $2008-10-07$ & 64 & 1.26 & 2.19 & 13.2 & $C$ & Grice et al. (2017) \\
\hline 81 & 2008-10-08 & 64 & 1.27 & 2.19 & 13.6 & $C$ & Grice et al. (2017) \\
\hline 82 & $2008-10-14$ & 47 & 1.29 & 2.18 & 15.9 & $C$ & Grice et al. (2017) \\
\hline 83 & $2008-10-14$ & 124 & 1.29 & 2.18 & 15.9 & $C$ & Grice et al. (2017) \\
\hline 84 & $2008-10-15$ & 38 & 1.30 & 2.17 & 16.3 & $C$ & Grice et al. (2017) \\
\hline 85 & $2008-10-16$ & 96 & 1.30 & 2.17 & 16.7 & $C$ & Grice et al. (2017) \\
\hline 86 & $2008-10-17$ & 41 & 1.31 & 2.17 & 17.0 & $C$ & Grice et al. (2017) \\
\hline 87 & $2008-10-18$ & 34 & 1.31 & 2.17 & 17.4 & $C$ & Grice et al. (2017) \\
\hline 88 & $2008-10-20$ & 92 & 1.33 & 2.17 & 18.1 & $C$ & Grice et al. (2017) \\
\hline 89 & $2008-10-23$ & 59 & 1.35 & 2.16 & 19.1 & $C$ & Grice et al. (2017) \\
\hline 90 & $2008-10-24$ & 28 & 1.35 & 2.16 & 19.4 & $C$ & Grice et al. (2017) \\
\hline 91 & $2008-10-24$ & 76 & 1.35 & 2.16 & 19.4 & $C$ & Grice et al. (2017) \\
\hline 92 & $2008-10-25$ & 30 & 1.36 & 2.16 & 19.8 & $C$ & Grice et al. (2017) \\
\hline 93 & $2008-10-26$ & 44 & 1.37 & 2.16 & 20.1 & $C$ & Grice et al. (2017) \\
\hline
\end{tabular}


Table B.3. continued.

\begin{tabular}{|c|c|c|c|c|c|c|c|}
\hline $\mathrm{N}$ & Epoch & $N_{\mathrm{p}}$ & $\begin{array}{c}\Delta \\
(\mathrm{AU})\end{array}$ & $\begin{array}{c}r \\
(\mathrm{AU})\end{array}$ & $\begin{array}{l}\varphi \\
\left({ }^{\circ}\right)\end{array}$ & Filter & Reference \\
\hline 94 & $2008-10-28$ & 29 & 1.38 & 2.16 & 20.7 & $C$ & Grice et al. (2017) \\
\hline 95 & 2008-10-31 & 35 & 1.40 & 2.15 & 21.6 & $C$ & Grice et al. (2017) \\
\hline 96 & 2008-11-01 & 40 & 1.41 & 2.15 & 21.8 & $C$ & Grice et al. (2017) \\
\hline 97 & $2008-11-03$ & 37 & 1.43 & 2.15 & 22.4 & $C$ & Grice et al. (2017) \\
\hline 98 & 2008-11-04 & 28 & 1.44 & 2.15 & 22.6 & $C$ & Grice et al. (2017) \\
\hline 99 & 2008-11-12 & 55 & 1.51 & 2.14 & 24.5 & $C$ & Grice et al. (2017) \\
\hline 100 & 2008-11-12 & 78 & 1.51 & 2.14 & 24.5 & $C$ & Grice et al. (2017) \\
\hline 101 & 2008-11-14 & 38 & 1.52 & 2.14 & 24.8 & $C$ & Grice et al. (2017) \\
\hline 102 & 2009-12-30 & 71 & 2.10 & 2.76 & 17.4 & $C$ & Grice et al. (2017) \\
\hline 103 & 2010-01-05 & 80 & 2.05 & 2.78 & 16.0 & $C$ & Grice et al. (2017) \\
\hline 104 & $2010-01-07$ & 31 & 2.04 & 2.78 & 15.5 & $C$ & Grice et al. (2017) \\
\hline 105 & 2010-01-10 & 25 & 2.02 & 2.79 & 14.7 & $C$ & Grice et al. (2017) \\
\hline 106 & 2010-01-11 & 60 & 2.01 & 2.79 & 14.5 & $C$ & Grice et al. (2017) \\
\hline 107 & 2010-01-12 & 27 & 2.00 & 2.79 & 14.2 & $C$ & Grice et al. (2017) \\
\hline 108 & 2010-01-13 & 41 & 2.00 & 2.80 & 13.9 & $C$ & Grice et al. (2017) \\
\hline 109 & 2010-01-14 & 26 & 1.99 & 2.80 & 13.7 & $C$ & Grice et al. (2017) \\
\hline 110 & $2010-01-15$ & 47 & 1.99 & 2.80 & 13.4 & $C$ & Grice et al. (2017) \\
\hline 111 & $2010-01-28$ & 60 & 1.93 & 2.83 & 9.6 & $C$ & Grice et al. (2017) \\
\hline 112 & 2010-02-02 & 122 & 1.92 & 2.85 & 8.3 & $C$ & Grice et al. (2017) \\
\hline 113 & $2010-02-03$ & 55 & 1.92 & 2.85 & 8.0 & $C$ & Grice et al. (2017) \\
\hline 114 & 2010-02-04 & 49 & 1.92 & 2.85 & 7.7 & $C$ & Grice et al. (2017) \\
\hline 115 & $2010-02-04$ & 78 & 1.92 & 2.85 & 7.8 & $C$ & Grice et al. (2017) \\
\hline 116 & 2010-02-05 & 45 & 1.92 & 2.86 & 7.5 & $C$ & Grice et al. (2017) \\
\hline 117 & $2010-02-05$ & 46 & 1.92 & 2.85 & 7.5 & $C$ & Grice et al. (2017) \\
\hline 118 & $2010-02-06$ & 29 & 1.92 & 2.86 & 7.3 & $C$ & Grice et al. (2017) \\
\hline 119 & 2010-02-06 & 34 & 1.92 & 2.86 & 7.3 & $C$ & Grice et al. (2017) \\
\hline 120 & 2010-02-07 & 35 & 1.92 & 2.86 & 7.1 & $C$ & Grice et al. (2017) \\
\hline 121 & 2010-02-08 & 22 & 1.92 & 2.86 & 6.9 & $C$ & Grice et al. (2017) \\
\hline 122 & 2010-02-08 & 25 & 1.92 & 2.86 & 6.9 & $C$ & Grice et al. (2017) \\
\hline 123 & 2010-02-09 & 48 & 1.92 & 2.87 & 6.7 & $C$ & Grice et al. (2017) \\
\hline 124 & $2010-02-10$ & 28 & 1.92 & 2.87 & 6.6 & $C$ & Grice et al. (2017) \\
\hline 125 & 2010-02-11 & 55 & 1.92 & 2.87 & 6.5 & $C$ & Grice et al. (2017) \\
\hline 126 & 2010-02-12 & 58 & 1.92 & 2.87 & 6.4 & $C$ & Grice et al. (2017) \\
\hline 127 & $2010-02-14$ & 58 & 1.92 & 2.88 & 6.3 & $C$ & Grice et al. (2017) \\
\hline 128 & $2010-02-15$ & 37 & & & & $C$ & Grice et al. \\
\hline 129 & $2010-02-15$ & 38 & 1.93 & 2.88 & 6.2 & $C$ & Grice et al. (2017) \\
\hline 130 & 2010-02-18 & 41 & 1.93 & 2.89 & 6.3 & $C$ & Grice et al. (2017) \\
\hline 131 & 2010-02-19 & 67 & 1.94 & 2.89 & 6.4 & $C$ & Grice et al. (2017) \\
\hline 132 & $2010-02-20$ & 30 & 1.94 & 2.89 & 6.5 & $C$ & Grice et al. (2017) \\
\hline 133 & $2010-02-23$ & 28 & 1.95 & 2.90 & 6.9 & $C$ & Grice et al. (2017) \\
\hline 134 & $2010-02-23$ & 34 & 1.95 & 2.90 & 6.9 & $C$ & Grice et al. (2017) \\
\hline 135 & $2010-02-24$ & 89 & 1.96 & 2.90 & 7.1 & $C$ & Grice et al. (2017) \\
\hline 136 & $2010-02-24$ & 90 & 1.96 & 2.90 & 7.1 & $C$ & Grice et al. (2017) \\
\hline 137 & 2010-03-01 & 32 & 1.98 & 2.91 & 8.1 & $C$ & Grice et al. (2017) \\
\hline 138 & $2010-03-02$ & 17 & 1.99 & 2.92 & 8.4 & $C$ & Grice et al. (2017) \\
\hline 139 & 2010-03-02 & 23 & 1.99 & 2.92 & 8.4 & $C$ & Grice et al. (2017) \\
\hline 140 & 2010-03-02 & 23 & 1.99 & 2.92 & 8.4 & $C$ & Grice et al. (2017) \\
\hline 141 & $2010-03-03$ & 44 & 2.00 & 2.92 & 8.6 & $C$ & Grice et al. (2017) \\
\hline 142 & 2010-03-03 & 46 & 2.00 & 2.92 & 8.6 & $C$ & Grice et al. (2017) \\
\hline 143 & 2010-03-03 & 53 & 2.00 & 2.92 & 8.6 & $C$ & Grice et al. (2017) \\
\hline 144 & 2010-03-10 & 45 & 2.05 & 2.94 & 10.5 & $C$ & Grice et al. (2017) \\
\hline 145 & $2010-03-13$ & 36 & 2.07 & 2.94 & 11.2 & $C$ & Grice et al. (2017) \\
\hline 146 & 2010-03-13 & 48 & 2.07 & 2.94 & 11.3 & $C$ & Grice et al. (2017) \\
\hline 147 & 2010-03-14 & 54 & 2.08 & 2.94 & 11.5 & $C$ & Grice et al. (2017) \\
\hline 148 & 2010-03-14 & 71 & 2.08 & 2.94 & 11.5 & $C$ & Grice et al. (2017) \\
\hline 149 & $2010-03-15$ & 32 & 2.09 & 2.95 & 11.7 & $C$ & Grice et al. (2017) \\
\hline 150 & $2010-03-16$ & 35 & & 2.95 & 12.0 & $C$ & Grice et al. (2017) \\
\hline 151 & 2010-03-17 & 53 & 2.11 & 2.95 & 12.3 & $C$ & Grice et al. (2017) \\
\hline 152 & $2010-03-18$ & 24 & 2.12 & 2.95 & 12.5 & $C$ & Grice et al. (2017) \\
\hline
\end{tabular}


Table B.3. continued.

\begin{tabular}{rcrcccll}
\hline \hline N & Epoch & $N_{\mathrm{p}}$ & $\begin{array}{c}\Delta \\
(\mathrm{AU})\end{array}$ & $\begin{array}{c}r \\
(\mathrm{AU})\end{array}$ & $\begin{array}{c}\varphi \\
\left({ }^{\circ}\right)\end{array}$ & Filter & Reference \\
\hline 153 & $2010-03-18$ & 43 & 2.12 & 2.95 & 12.5 & $C$ & Grice et al. (2017) \\
154 & $2010-03-19$ & 15 & 2.13 & 2.96 & 12.8 & $C$ & Grice et al. (2017) \\
155 & $2010-03-20$ & 50 & 2.14 & 2.96 & 13.0 & $C$ & Grice et al. (2017) \\
156 & $2010-03-20$ & 96 & 2.14 & 2.96 & 13.0 & $C$ & Grice et al. (2017) \\
157 & $2010-03-21$ & 33 & 2.15 & 2.96 & 13.2 & $C$ & Grice et al. (2017) \\
158 & $2010-03-21$ & 36 & 2.15 & 2.96 & 13.3 & $C$ & Grice et al. (2017) \\
159 & $2010-03-22$ & 23 & 2.16 & 2.96 & 13.5 & $C$ & Grice et al. (2017) \\
160 & $2010-03-22$ & 40 & 2.16 & 2.96 & 13.5 & $C$ & Grice et al. (2017) \\
161 & $2010-03-23$ & 64 & 2.18 & 2.97 & 13.7 & $C$ & Grice et al. (2017) \\
162 & $2010-03-24$ & 26 & 2.19 & 2.97 & 13.9 & $C$ & Grice et al. (2017) \\
163 & $2010-03-24$ & 62 & 2.19 & 2.97 & 14.0 & $C$ & Grice et al. (2017) \\
164 & $2010-03-29$ & 55 & 2.25 & 2.98 & 15.0 & $C$ & Grice et al. (2017) \\
165 & $2010-03-29$ & 116 & 2.24 & 2.98 & 15.0 & $C$ & Grice et al. (2017) \\
166 & $2010-03-30$ & 50 & 2.26 & 2.98 & 15.2 & $C$ & Grice et al. (2017) \\
167 & $2010-03-30$ & 70 & 2.26 & 2.98 & 15.2 & $C$ & Grice et al. (2017) \\
168 & $2010-03-31$ & 61 & 2.27 & 2.98 & 15.4 & $C$ & Grice et al. (2017) \\
169 & $2010-04-01$ & 67 & 2.28 & 2.99 & 15.6 & $C$ & Grice et al. (2017) \\
170 & $2010-04-04$ & 41 & 2.32 & 2.99 & 16.1 & $C$ & Grice et al. (2017) \\
171 & $2010-04-06$ & 24 & 2.35 & 3.00 & 16.5 & $C$ & Grice et al. (2017) \\
172 & $2010-04-07$ & 40 & 2.36 & 3.00 & 16.6 & $C$ & Grice et al. (2017) \\
173 & $2010-04-08$ & 37 & 2.37 & 3.00 & 16.8 & $C$ & Grice et al. (2017) \\
174 & $2010-04-10$ & 39 & 2.40 & 3.01 & 17.1 & $C$ & Grice et al. (2017) \\
175 & $2010-04-12$ & 35 & 2.43 & 3.01 & 17.4 & $C$ & Grice et al. (2017) \\
176 & $2011-02-20$ & 9 & 2.96 & 3.47 & 15.2 & $C$ & Grice et al. (2017) \\
177 & $2013-10-23$ & 200 & 1.18 & 2.11 & 13.1 & $C$ & Grice et al. (2017) \\
178 & $2013-10-28$ & 174 & 1.16 & 2.11 & 10.7 & $C$ & Grice et al. (2017) \\
179 & $2013-10-30$ & 115 & 1.15 & 2.11 & 9.7 & $C$ & Grice et al. (2017) \\
180 & $2015-04-15.0$ & 245 & 2.41 & 3.25 & 11.4 & $R$ & This work \\
\hline
\end{tabular}


Table B.4. Individual observers that participated in the stellar occultation campaigns targeting asteroid Kleopatra (Herald et al. 2020).

Observer

1980-10-10

S. Krysko, Linbrook, Alberta

G. Stokes, Richland, WA

Beals/Belcher/Loehde, Cooking Lake

D. Scarlett, Kaslo, B.C.

Jones/Bowen, Castlegar, B.C.

D. Hube, Devon, U. of Alberta, AL

G. Fouts, Goldendale, WA

Mitchell, Ellensburg, WA

E. Mannery, Ellensburg, WA

$$
\text { 1991-01-19 }
$$

D. Dunham/W. Warren, Maryland, NJ Jeff Guerber, Greenbelt, MD

C. Aikman/M. Fletcher, Victoria, BC

Robert J. Mordic, Richmond Hts, $\mathrm{OH}$

Samuel Storch, Jones Beach, NY

Jim Pyral, North Bend, WA

Edwin Lurcott, West Chester, PA

M. Henry, Canton, $\mathrm{OH}$

James H. Fox, Afton, MN

Robert Bolster, Alexandria, VA

Dan Grieser, Columbus, $\mathrm{OH}$

$$
\text { 2009-12-24 }
$$

D. Dunham et al, Piedra, AZ

J. Ray, Glendale, AZ

R. Peterson, Phoenix, AZ

R. Peterson, Scottsdale, AZ

P. Maley, Sun Lakes, AZ

G. Rattley, Gilbert, AZ

P. Maley, Santan, AZ

S. Degenhardt, Quijotoa, AZ

L. Martinez, Casa Grande, AZ

S. Degenhardt, Gu Oldak, AZ

S. Degenhardt, Sells, AZ

S. Degenhardt, Ali Chukson, AZ

S. Degenhardt, Schuchk, AZ

S. Degenhardt, Three Points, AZ

J. Stamm, Oro Valley, AZ

$$
\text { 2015-03-12 }
$$

Henk Bulder, NL

Friedhelm Dorst, DE

Oliver Kloes, DE

Jan-Maarten Winkel, NL

Otto Farago, DE

Vasilis Metallinos, GR

Harrie Rutten, NL

Henk De Groot, NL

Bernd Gaehrken, DE

Hans Kostense, NL

D. Fischer, H.G. Purucker, R. Stoyan, DE

Eberhard Bredner, FR

Andre Mueller, DE
Table B.4. continued.

\begin{tabular}{l}
\hline \hline Observer \\
\hline Lex Blommers, NL \\
K. Moddemeijer, P. Bastiaansen, W. Nobel, NL \\
Christof Sauter, CH \\
Maxime Devogele, BE \\
Mike Kohl, CH \\
Jose De Queiroz, CH \\
Karl-Ludwig Bath, DE \\
Martin Federspiel, DE \\
Fernand Emering, LU \\
F. Van Den Abbeel, BE \\
Rene Bourtembourg, BE \\
Jonas Schenker, CH \\
J. Lecacheux, E. Meza, FR \\
Stefano Sposetti, CH \\
Roberto Di Luca, IT \\
T. Pauwels, P. De Cat, BE \\
C. Demeautis. D. Matter, FR \\
Stefano Sposetti, CH \\
Andrea Manna, CH \\
Alberto Ossola, CH \\
Carlo Gualdoni, IT \\
Roland Decellier, BE \\
Fabrizio Ciabattari, IT \\
Mauro Bachini, IT \\
Giancarlo Bonatti, IT \\
Alex Pratt, UK \\
Gilles Sautot, FR \\
Roland Boninsegna, BE \\
Fausto Delucchi, CH \\
Martin Federspiel, DE \\
G. Sautot, E. Vauthrin, FR \\
Olivier Dechambre, FR \\
Jerome Berthier, FR \\
Frederic Vachier, FR \\
B. Carry, M. Pajuelo, FR \\
Joan Rovira, ES \\
B. Dunford, Naperville, IL \\
A. Olsen, Urbana, IL \\
D. Dunham/J. Dunham, Yemasse, SC \\
D. Dunham/J. Dunham, Coosawhatchie, SC \\
D. Dunham/J. Dunham, Hardeesville, SC \\
D. Dunham/J. Dunham, Savannah, GA \\
D. Dunham/J. Dunham, Midway, GA \\
D. Dunham/J. Dunham, South Newport, GA \\
D. Dunham/J. Dunham, Darien, GA \\
N. Smith, Trenton, GA \\
R. Venable, Yonkers, GA \\
R. Venable, Hawkinsville, GA \\
S. Messner, Moravia, IA \\
S. Messner, Iconium, IA \\
R. Venable, Oakfield, GA \\
R. Venable, Newton, GA \\
\hline
\end{tabular}

